\title{
REVISITING CAUTY'S PROOF OF THE SCHAUDER CONJECTURE
}

\author{
TADEUSZ DOBROWOLSKI
}

Received 18 April 2002

The Schauder conjecture that every compact convex subset of a metric linear space has the fixed-point property was recently established by Cauty (2001). This paper elaborates on Cauty's proof in order to make it more detailed and therefore more accessible. Such a detailed analysis allows us to show that the convex compacta in metric linear spaces possess the simplicial approximation property introduced by Kalton, Peck, and Roberts. The latter demonstrates that the original Schauder approach to solve the conjecture is in some sense "correctable."

\section{Introduction}

Throughout most of this paper, $X$ will be a compact convex subset of a separable metric linear space $(E,\|\cdot\|)$ which is not necessarily locally convex. We can always assume (and we will) that $\|\cdot\|$ is an $F$-norm on $E$; hence, we have $\|x+y\| \leq\|x\|+\|y\|,\|t x\| \leq\|x\|$ for all $x, y \in E$ and $-1 \leq t \leq 1$; in general, $\|\cdot\|$ is not homogeneous. Generalizing the classical Brouwer theorem, in 1930, Schauder [28] claimed the proof of the fixed-point property of $X$; unfortunately, his argument contained a gap. As an effect, the question of whether every compact convex subset of a metric linear space has the fixed-point property was put, in August 1935, [25, Problem 54]; it became known as Schauder's conjecture. Since then, many partial results were obtained but the general case went unsettled. Almost all those partial results were based on the so-called finitedimensional approximation property that some convex compacta possess. The property requires that, $\mathrm{id}_{X}$, the identity map on $X$, is, in a uniform way, arbitrarily closely approximated by maps $\psi: X \rightarrow X$ such that $\operatorname{dim}(\psi(X))<\infty$. Here, the dimension "dim" can be understood in any reasonable (i.e., "linear" or purely topological) way; for further information, see [7]. In the case that $E$ is locally convex, such an approximation can be obtained via partitions of unity. Such an 
approach predominates in the textbook proofs of the Schauder-Tychonoff theorem which states that every compact convex subset of a locally convex topological vector space has the fixed-point property. This most transparent fact in the area was published by Tychonoff [29] in 1935.

Note that, as a consequence of some advanced results of infinite-dimensional topology, a convex compactum $X$ having the finite-dimensional property not only has the fixed-point property, but actually is homeomorphic either to $[0,1]^{n}$, $n \in \mathbb{N}$, or to $[0,1]^{\infty}$, the Hilbert cube, see $[7,12]$. As later becomes clear, it is remarkable that Schauder in his failed attempt did not intend to verify the finite-dimensional property, but a weaker property which was later formalized by Kalton et al. in [21]. This "local" property, which is called the simplicial approximation property in [21], yields the fixed-point property, but itself is rather far from the finite-dimensional property, which in turn could be viewed as a "global" property. In [21], the simplicial approximation property was used to verify the fixed-point property of certain convex compacta without extreme points. Those compacta were earlier discovered by Roberts.

Finally, in 1999, Cauty [5] provided a proof of the Schauder conjecture. His proof did not rely on any of the above properties, but was based on the existence of a certain resolution map $\varphi: Z \rightarrow X$ from a certain countable-dimensional compactum $Z$ onto $X$. Actually, Cauty has proved a more general result stating that, for an arbitrary convex subset $C$ of a topological vector space, every map $f: C \rightarrow C$ such that $f(C)$ is contained in a compact subset of $C$ (i.e., every relatively compact map $f: C \rightarrow C$ ) has the fixed-point property. We mention that a similar result for the locally convex case was obtained by Mazur [26] and Hukuhara [19] in 1938 and 1950, respectively.

This paper elaborates on Cauty's proof of the metric case only; that is, on the following result.

TheOREM 1.1 (Cauty [5]). Let $X$ be a convex compactum of a metric linear space $(E,\|\cdot\|)$ and let $f: X \rightarrow X$ be a map. There exists $x \in X$ with $f(x)=x$.

Revisiting Cauty's proof, we were able to isolate its two basic ingredients. The first ingredient is of purely topological (or, better to say, metric) nature; it deals with the construction of the resolution $\varphi$. The second ingredient has a linear (affine) flavor and, employing the resolution map $\varphi$, it establishes a certain approximation property. We hope that our analysis makes Cauty's proof more accessible. Surprisingly, such an analysis enables us to verify the simplicial approximation property of $X$ (cf. [8]). This shows that the original approach of Schauder is "correctable." As it stands, the proof of the fixed-point property is very much complex. Now, knowing that the simplicial approximation property holds for every $X$, it is reasonable to ask for a simple way of verifying it.

According to a result of [10], every noncompact convex subset $C$ of a metric linear space contains a topological copy of $[0, \infty)$ (this observation, for the normed case, was made long before by Klee [22]). As easily observed, it follows 
that such a $C$ fails the fixed-point property. Combining this with Cauty's result, we infer that for convex subsets of metric linear spaces, only compacta have the fixed-point property. On the other hand, there are examples of noncompact convex subsets of locally convex topological vector spaces that do have the fixedpoint property, see [10, Example 4.1].

Cauty has extended his technique to show the fixed-point property for compacta that are uniformly contractible. Recall that a space $X$ is locally uniformly contractible if there exists a continuous map $\mu(x, y ; t)$ (which is referred to as an equiconnecting map) defined for all $(x, y)$ in a neighborhood $U$ of the diagonal in $X \times X$ and $t \in I=[0,1]$, such that $\mu(x, y ; 0)=x, \mu(x, y ; 1)=y$, and $\mu(x, x ; t)=$ $x$ for all $(x, y ; t) \in U \times I$. If $U$ can be taken as the whole $X \times X$, then $X$ is called uniformly contractible. All convex sets are uniformly contractible. If $r: C \rightarrow X$ is a retraction of a convex set $C$ onto $X$, then $X$ is uniformly contractible because $\mu(x, y ; t)=r((1-t) x+t y)$ is as required. If a topological group $G$ is contractible, that is, there exists a homotopy $H: G \times I \rightarrow G$ such that $H(g, 0)=g$ and $H(g, 1)=e(e$ is the unit element of $G$ ) for all $(g, t) \in G \times I$, then $G$ is uniformly contractible via $\mu(g, h ; t)=(H(e, t))^{-1} \cdot H\left(g \cdot h^{-1}, t\right) \cdot h$, see [7]. It follows that every retract of such a $G$ is uniformly contractible. On the other hand, a metrizable uniformly contractible compactum is a retract of a contractible metrizable topological group [3]. Generalizing the classical Lefschetz-Hopf fixed-point theorem, Cauty [4] has shown that every self-map $f$ of a locally uniformly contractible compactum $X$ has a fixed-point if the Lefschetz number $\Lambda(f) \neq 0$. In particular, all uniformly contractible compacta have the fixed-point property. Necessary modifications for obtaining the proof of this fact are presented at the end of Section 2 (see also the end of Section 3). Observe that every uniformly contractible compactum is a contractible and locally contractible space. The question of whether a contractible and locally contractible metrizable compactum has the fixed-point property remains unanswered.

For the locally convex case, the fixed-point property of $X$ yields, for an upper semicontinuous (USC) convex-valued map $F: X \Rightarrow X$, the fixed-point property of $F$, that is, there exists $x \in X$ such that $x \in F(x)$. (Here, by a convex-valued map $F$, we mean a multivalued map such that $F(x)$ is a convex compactum; such a map is USC if $\{x \in X \mid F(x) \subset U\}$ is open for every open set $U \subset X$.) Again, known techniques require partitions of unity, a tool that does not work for the nonlocally convex case. Our approach enabled us to show that, for every dense convex subset $C$ of the convex compactum $X$, every map $f: C \rightarrow X$ admits approximate fixed-points; that is, there exists a sequence $\left(x_{n}\right) \subset C$ such that $\lim \left\|f\left(x_{n}\right)-x_{n}\right\|=0$, see Corollary 2.6. In [9], we used this fact, together with yet another approximation result that was based on a certain technique developed by Cellina-Lasota [6], to obtain the fixed-point property for every USC convex-valued map $F$ of a compact convex set of an arbitrary topological vector space. The details of such a generalization of Cauty's result go beyond the scope of this paper and will not be included here. For the record, we mention that 
the convex-valued case has been established, among others, by Kakutani [20], Bohnenblust and Karlin [2], Fan [15], and Glicksberg [16].

This introduction alludes to the author's observations that were made while revisiting Cauty's paper [5]. It contains only a handful of historical remarks on the fixed-point property of convex compacta that were needed to put those observations in some historical perspective. The author did not intend to make an expository paper on the subject with such overwhelming literature.

\section{The simplicial approximation property}

Following [21], we say that a convex set $C \subset E$ has the simplicial approximation property if for every $\varepsilon>0$, there exists a finite-dimensional compact convex set $C_{\varepsilon} \subset C$ such that if $S$ is any finite-dimensional simplex in $C$ (i.e., $S$ is a convex hull of finitely many vectors in $C$ ), then there exists a continuous map $\gamma: S \rightarrow C_{\varepsilon}$ with $\|\gamma(x)-x\|<\varepsilon, x \in S$. According to [21, Remark (2), page 217] (see also [11, Lemma 5.1]), if a convex compactum $X$ has the simplicial approximation property, then it has the fixed-point property. We have the following obvious generalization of that fact.

Lemma 2.1. Assume that a convex set $C \subset E$ has the simplicial approximation property. Then every map $f: C \rightarrow \bar{C}$ has an approximate fixed-point, that is, $\lim$ $\left\|f\left(x_{n}\right)-x_{n}\right\|=0$ for some sequence $\left(x_{n}\right) \subset C$.

Proof. According to the definition above, for each $n \in \mathbb{N}$, pick a convex compactum $C_{n} \subset C$ such that for a simplex $S$, we have a sequence $\gamma_{n}: S \rightarrow C_{n}$ with $\lim \left\|\gamma_{n}-\operatorname{id}_{S}\right\|=0$. Approximate $f \mid C_{n}$ by a map $f_{n}: C_{n} \rightarrow S_{n}$, where $S_{n}$ is a simplex in $C$, see [7]. By Brouwer fixed-point theorem, there exists $x_{n} \in C_{n}$ such that $\gamma_{n}\left(f_{n}\left(x_{n}\right)\right)=x_{n}$. It is easy to see that $\lim \left\|f\left(x_{n}\right)-x_{n}\right\|=0$.

Say that a convex set $C \subset E$ has the $\mathrm{N}_{0}$-simplicial approximation property if for every $\varepsilon>0$, there exists a countable-dimensional convex set $C_{\varepsilon} \subset C$ such that if $S$ is any $\mathrm{N}_{0}$-simplex in $C$ (i.e., $S$ is a convex hull of countable many vectors of $C)$, then there exists a relatively compact map $\gamma: S \rightarrow C_{\varepsilon}$ with $\|\gamma(x)-x\|<\varepsilon$, $x \in S$. Actually, this property is equivalent to the one that requires $C_{\varepsilon}$ to be an $\aleph_{0}$-simplex. To see this, use the separability of $E$ to find an $\aleph_{0}$-simplex $C_{\varepsilon}^{\prime} \subset C_{\varepsilon}$ that is dense in $C_{\varepsilon}$. Now, let $\gamma$ be a relatively compact map of $S$ into $C_{\varepsilon}$. Using the fact that $C_{\varepsilon}$ is countable dimensional, find a map of $C_{\varepsilon}$ into $C_{\varepsilon}^{\prime}$ that is as close to the identity as we wish, see [7]. Composing this map with $\gamma$, we obtain a relatively compact map of $S$ into $C_{\varepsilon}^{\prime}$.

Lemma 2.2. If a convex set $C \subset E$ has the $\mathrm{N}_{0}$-simplicial approximation property, then it has the simplicial approximation property.

Proof. Fix $\varepsilon>0$. Use the above modification of the $\mathrm{N}_{0}$-simplex approximation property to find, for $\varepsilon / 3$, an $\mathrm{N}_{0}$-simplex $C_{\varepsilon} \subset C$ as required in the definition. Let 
$\gamma_{0}: C_{\varepsilon} \rightarrow C_{\varepsilon}$ be a relatively compact map with $\left\|\gamma_{0}(x)-x\right\|<\varepsilon / 3, x \in C_{\varepsilon}$. There exists a map $g: \gamma_{0}\left(C_{\varepsilon}\right) \rightarrow C_{\varepsilon}$ whose range is contained in a finite-dimensional compact convex set $K_{\varepsilon}$ and that satisfies $\|g(x)-x\|<\varepsilon / 3$ for all $x$, see [7, Lemma 2]. Now, let $S$ be a finite-dimensional simplex in $C$. Assuming that $C_{\varepsilon}$ is dense (here we employ the separability of $E$ ), we find a map $\gamma: S \rightarrow C_{\varepsilon}$ with $\| \gamma(x)-$ $x \|<\varepsilon / 3, x \in S$. Then, the composition $g \circ \gamma_{0} \circ \gamma: S \rightarrow K_{\varepsilon}$ is as required.

Remark 2.3. Replacing the convexity assumption on $C_{\varepsilon}$ by the assumption that $C_{\varepsilon} \in \mathrm{ANR}$, we obtain a weaker version of the $\mathrm{N}_{0}$-simplicial approximation property. Such a weaker property also yields the approximate fixed-point property of $C$. To see this, we employ the ANR property of $C_{\varepsilon}$ (use a factorization of an ANR through a locally finite-dimensional, locally compact, separable space) to approximate a map $C_{\varepsilon} \rightarrow C$ by one whose range is $S$, an $\aleph_{0}$-simplex. Next, using the contractibility of $S$, we extend this map to $c\left(C_{\varepsilon}\right)$, the metric cone over $C_{\varepsilon}$. Composing such a map with a relatively compact $\gamma: S \rightarrow C_{e} \subset c\left(C_{\varepsilon}\right)$, which is provided by the definition (of such a weaker version of the $\mathrm{N}_{0}$-simplicial approximation property), we obtain a relatively compact self-map of $c\left(C_{\varepsilon}\right)$. An application of the so-called generalized Schauder theorem (stating that every relatively compact self-map of an AR space has the fixed-point property [13, page 94]) yields a fixed-point $x_{e} \in C_{e}$, which is a counterpart of $x_{n}$ that was obtained in the proof of Lemma 2.1.

Below, we state the main technical ingredient of Cauty's proof that establishes a property which, in a sense, is equivalent to the $\mathrm{N}_{0}$-simplicial approximation property of $X$.

Proposition 2.4 (cf. [5, Lemma 3]). For the convex compactum $X$, there exist a countable-dimensional compactum $Z$ and a map $\varphi: Z \rightarrow X$ such that, if $Z$ is embedded onto a linearly independent subset of a metric linear space $(F,|\cdot|)$ so that the affine extension $\hat{\varphi}: \operatorname{conv}(Z) \rightarrow X$ of $\varphi$ is continuous (the existence of such an embedding is ensured by Lemma 2.5 below), then, for every $\varepsilon>0$, every separable metrizable space $Y \in \mathrm{ANR}$, and every map $\xi: Y \rightarrow X$, there exists a map

$$
\eta: Y \longrightarrow \operatorname{conv}(Z)
$$

such that

(i) $\|\hat{\varphi}(\eta(y))-\xi(y)\|<\varepsilon$ for all $y \in Y$,

(ii) $\eta(Y) \cup \operatorname{conv}_{2}(Z)$ is a compact subset of $\operatorname{conv}(Z)$; hence, $\eta$ is relatively compact.

Here by $\operatorname{conv}_{2}(Z)$, we mean $\left\{t_{1} z_{1}+t_{2} z_{2} \mid z_{1}, z_{2} \in Z, 0 \leq t_{1}, t_{2} \leq 1, t_{1}+t_{2}=1\right\}$.

Lemma 2.5. Given a map $\varphi: Z \rightarrow X$ of a compactum $Z$, there exists a metric linear space $(F,|\cdot|)$ topologically containing $Z$ as a linearly independent subset such that 
412 Revisiting Cauty's proof of the Schauder conjecture

$\hat{\varphi}: \operatorname{conv}(Z) \rightarrow X$ defined by

$$
\hat{\varphi}\left(\sum_{i=1}^{k} t_{i} z_{i}\right)=\sum_{i=1}^{k} t_{i} \varphi\left(z_{i}\right),
$$

where $\sum_{i=1}^{k} t_{i}=1, t_{i} \geq 0, k \in \mathbb{N}$, is continuous.

Proof of Lemma 2.5. Embed $Z$ onto a linearly independent subset of $\left(\ell_{2},|\|\cdot\||\right)$. Extend $\varphi$ to a linear (not necessarily continuous) map $\hat{\varphi}: \ell_{2} \rightarrow E$. Define an $F$ norm on $\ell_{2}$ by

$$
|x|=|\|x\||+\|\hat{\varphi}(x)\| .
$$

Let $F=\left(\ell_{2},|\cdot|\right)$. Then $\hat{\varphi}: F \rightarrow E$ is continuous, and $(Z,|\cdot|)$ yields the original topology of $Z$.

Corollary 2.6 (cf. [8, Theorem]). Every convex set $C \subset E$ with a compact closure $X$ has the $\mathrm{N}_{0}$-simplicial approximation property. Consequently, by Lemma 2.1, every map $f: C \rightarrow X$ has an approximate fixed-point.

Proof. Let $Z, \varphi$, and $\hat{\varphi}$ be that of Proposition 2.4. Fix $\varepsilon>0$. Since the compactum $Z$ is countable dimensional, so is $\operatorname{conv}(Z)$. An application of an argument of [7] shows that there exist an $\aleph_{0}$-simplex $X_{\varepsilon} \subset C$ and a map $\varphi_{\varepsilon}: \operatorname{conv}(Z) \rightarrow X_{\varepsilon}$ such that $\left\|\hat{\varphi}(z)-\varphi_{\varepsilon}(z)\right\|<\varepsilon / 2, z \in \operatorname{conv}(Z)$. Let $S$ be $\kappa_{0}$-simplex in $C$. By a theorem of Haver [17] (see also [7, Note 4]), we have $S \in$ AR. Apply Proposition 2.4 to $Y=S$ and to $\xi$, the inclusion of $S$ into $X$. There exists a relatively compact $\eta$ : $S \rightarrow \operatorname{conv}(Z)$ with $\|\hat{\varphi} \circ \eta(x)-x\|<\varepsilon / 2, x \in S$. Let $\gamma=\varphi_{\varepsilon} \circ \eta: X \rightarrow X_{\varepsilon}$. We have that $\|\gamma(x)-x\| \leq\left\|\varphi_{\varepsilon} \circ \eta(x)-\hat{\varphi} \circ \eta(x)\right\|+\|\hat{\varphi} \circ \eta(x)-x\| \leq \varepsilon / 2+\varepsilon / 2=\varepsilon$.

Proof of Theorem 1.1. Applying Corollary 2.6, we conclude that $f$ has an approximate fixed-point. By the compactness of $X, f$ has a fixed-point.

Reduction Fact 2.7. The assertion of Proposition 2.4 holds provided it does hold for the class of spaces $Y$ that are separable, metrizable, locally finite dimensional, and locally compact.

Proof. Let $Y$ be an arbitrarily separable, metrizable ANR, let $\xi: Y \rightarrow X$ be a map, and let $\varepsilon>0$. By [18, page 138], there exist a countable locally finite simplicial complex $|N|$ (considered in the metric topology) and maps

$$
\alpha: Y \longrightarrow|N|, \quad \beta:|N| \longrightarrow Y
$$

such that

$$
\|\xi(\beta \circ \alpha(y))-\xi(y)\|<\frac{\varepsilon}{2},
$$


$y \in Y$. The space $|N|$ is separable, locally finite dimensional, and locally compact. For the map $\xi \circ \beta:|N| \rightarrow X$, we can find a relatively compact map $\tilde{\eta}:|N| \rightarrow$ $\operatorname{conv}(Z)$ with $\|\hat{\varphi} \circ \tilde{\eta}(p)-\xi \circ \beta(p)\|<\varepsilon / 2, p \in|N|$. Set $\eta=\tilde{\eta} \circ \alpha: Y \rightarrow \operatorname{conv}(Z)$. We see that $\eta$ is relatively compact and $\|\hat{\varphi} \circ \eta(y)-\xi(y)\|=\| \hat{\varphi} \circ \tilde{\eta}(\alpha(y))-\xi(\beta \circ$ $\alpha(y))\|+\| \xi(\beta \circ \alpha(y))-\xi(y) \|<\varepsilon / 2+\varepsilon / 2=\varepsilon$.

Since every map of a countable locally finite simplicial metric complex into $X$ can be uniformly, arbitrarily closely approximated by a map whose range is contained in an $\kappa_{0}$-simplex $S$, Proposition 2.4 holds if we verify its assertion for all $Y=S$, where $S$ is such a simplex. In this way, Proposition 2.4 establishes a property that is equivalent to the $\mathrm{N}_{0}$-simplicial approximation property. A direct argument for the $\mathrm{N}_{0}$-simplicial approximation property in case $X$ enjoys the simplicial approximation property is not known to us. Also the relationship of the finite-dimensional approximation property to both simplicial approximation properties is unclear.

The results of Proposition 2.4, Lemma 2.5, and Reduction Fact 2.7 can be extended to the uniformly contractible spaces $X$; consequently, the fixed-point property holds for such $X$. We say that $\psi: \operatorname{conv}(Z) \rightarrow X$ is $\mu$-affine ( $\mu$ is an equiconnecting map $X)$ if $\mu\left(\psi\left(z_{1}\right), \psi\left(z_{2}\right) ; t\right)=(1-t) z_{1}+t z_{2}$ for all $z_{1}, z_{2} \in \operatorname{conv}(Z)$ and $t \in I$. Proposition 2.4 holds for a uniformly contractible compactum $X$ if $\varphi$ admits a continuous $\mu$-affine extension $\hat{\varphi}: \operatorname{conv}(Z) \rightarrow X$, where $\operatorname{conv}(Z)$ is a convex subset (of a vector space) with a metric topology that makes the convex combination map $\left(z_{1}, z_{2} ; t\right) \rightarrow(1-t) z_{1}+t z_{2}$ continuous. Such an extension can be obtained by inspecting the proof of Lemma 2.5. As in that proof, we embed the compactum $Z$ onto a linearly independent subset of $\ell_{2}$. Next, extend $\varphi$ to (not necessarily continuous) $\hat{\varphi}: \operatorname{conv}(Z) \rightarrow X$ that is $\mu$-affine. Finally, letting $d$ for a metric on $X, \rho\left(z_{1}, z_{2}\right)=\left|\left\|z_{1}-z_{2}\right\|\right|+d\left(\hat{\varphi}\left(z_{1}\right), \hat{\varphi}\left(z_{2}\right)\right)$ defines a required metric on $\operatorname{conv}(Z)$, a convex subset of $\ell_{2}$. Before we show how to obtain $\hat{\varphi}$, for $x_{1}, \ldots, x_{n} \in X$ and $\left(t_{1}, \ldots, t_{n}\right) \in s_{n}=\left\{\left(t_{1}, \ldots, t_{n}\right) \mid t_{i} \geq 0\right.$ for all $i$ and $\left.\sum_{i=1}^{n} t_{i}=1\right\}$, we inductively let

$$
\mu_{n}\left(x_{1}, \ldots, x_{n} ; t_{1}, \ldots, t_{n}\right)=\mu_{n-1}\left(x_{1}, \ldots, x_{n-1} ; t_{1} /\left(1-t_{n}\right), \ldots, t_{n-1} /\left(1-t_{n}\right)\right)
$$

if $t_{n} \neq 1$, and $\mu_{n}\left(x_{1}, \ldots, x_{n} ; t_{1}, \ldots, t_{n}\right)=x_{n}$ otherwise; set $\mu_{1}\left(x_{1} ; 1\right)=x_{1}$. Now, well order the set $Z$ by a relation $<$ and, for $z=\sum_{i=1}^{n} t_{i} z_{i}$ where $\left(t_{1}, \ldots, t_{n}\right) \in s_{n}$ and $z_{1}, \ldots, z_{n} \in Z$ with $z_{1}<\cdots<z_{n}$, define $\hat{\varphi}(z)=\mu_{n}\left(\varphi\left(z_{1}\right), \ldots, \varphi\left(z_{n}\right) ; t_{1}, \ldots, t_{n}\right)$. It is easily seen that $\hat{\varphi}$ is $\mu$-affine. Using the cross-section method (see [1, page $271])$, we can show that $\operatorname{conv}(Z)=(\operatorname{conv}(Z), \rho)$ is countable dimensional. Since $\operatorname{conv}(Z)$ is locally contractible and contractible, by a theorem of Haver [17], $\operatorname{conv}(Z) \in$ AR. To conclude the fixed-point property of $X$ by such a generalized version of Proposition 2.4, we find a sequence of relatively compact maps $\left(\eta_{n}\right)$ of $Y=\operatorname{conv}(Z)$ with $\lim \hat{\varphi} \circ \eta_{n}=f \circ \hat{\varphi}$. By a generalized Schauder theorem $\left[13\right.$, page 94], there exists $y_{n} \in Y$ such that $\eta\left(y_{n}\right)=y_{n}$. We can assume that the 
sequence $\left(\hat{\varphi}\left(y_{n}\right)\right)$ converges to $x \in X$. Such an $x$ is a fixed-point of $f$ because $f(x)=\lim f\left(\hat{\varphi}\left(y_{n}\right)\right)=\lim \hat{\varphi}\left(\eta_{n}\left(y_{n}\right)\right)=\lim \hat{\varphi}\left(y_{n}\right)=x$.

\section{The proof of Proposition 2.4}

This section contains the proof of Proposition 2.4. The proof will rely on Lemma 3.1, a purely topological fact, which is an abstraction on Cauty's construction of the resolution $\varphi: Z \rightarrow X$ (that will be provided in Section 4). However, after completing the proof of Proposition 2.4, we give a rough sketch of the proof of Lemma 3.1 for the reader who is not interested in all details.

Lemma 3.1. Let $(X, d)$ be a metric space. There exist an inverse sequence $\left(Z_{n}, \pi_{n}^{n+1}\right)$, where each $Z_{n}$ is a (finite-dimensional) compactum, a map $k_{n}: X \rightarrow Z_{n}$, a finite open cover $\left\{W_{\alpha} \mid \alpha \in A(n)\right\}$ of $Z_{n}, n \in \mathbb{N}$, and a map $\varphi: Z=\underline{\lim }\left(Z_{n}, \pi_{n}^{n+1}\right) \rightarrow X$ that satisfy the following conditions:

(1) $Z$ is a countable-dimensional compactum;

(2) $d\left(\varphi(z), \bar{V}_{\alpha}\right) \leq 2 \Delta_{n}$ whenever $\pi_{n}(z) \in W_{\alpha}$ and $\alpha \in A(n)$, where $\pi_{n}: Z \rightarrow Z_{n}$ is an obvious projection and $V_{\alpha}=k_{n}^{-1}\left(W_{\alpha}\right), \alpha \in A(n)$;

(3) $\Delta_{n}=\sup \left\{\operatorname{diam}_{d}\left(V_{\alpha}\right) \mid \alpha \in A(n)\right\} \rightarrow \infty$ as $n \rightarrow \infty$.

The space $Z=\lim \left(Z_{n}, \pi_{n}^{n+1}\right)$ is equipped with a metric $d_{Z}$, a restriction of $d_{Z}\left(\left(x_{n}\right),\left(y_{n}\right)\right)=\sum_{n=1}^{\infty} 2^{-n} d_{n}\left(x_{n}, y_{n}\right)$ defined on $\prod_{n=1}^{\infty} Z_{n}$, where each $d_{n}$ is a compatible, bounded by 1 , metric on $Z_{n}$.

Proof of Proposition 2.4. The convex compactum $X$ will be equipped with the metric $d$ that is induced by an $F$-norm $\|\cdot\|$. Lemma 3.1 provides us with a compactum $Z$ and a map $\varphi: Z \rightarrow X$ that satisfy (1), (2), and (3). (Further on, the countable dimensionality of $Z$ will not be used.) Moreover, $Z$ is assumed to be a linearly independent subset of a metric linear space $(F,|\cdot|)$ such that the affine extension $\hat{\varphi}: \operatorname{conv}(Z) \rightarrow X$ is continuous. Let $\xi: Y \rightarrow X$ be a map, and let $\varepsilon>0$, $\varepsilon \leq 1$. Applying Reduction Fact 2.7, we may assume that $Y$ is a locally finitedimensional, locally compact, separable, metrizable space. There exists an open cover $\left\{Y_{i}\right\}_{i=1}^{\infty}$ such that, for every $i, Y_{i}$ is relatively compact, $\operatorname{dim}\left(Y_{i}\right) \leq i$, and $Y_{i} \cap Y_{j}=\varnothing$ whenever $|i-j|>1$, see [14, page 291].

By the compactness of $Z$, for each $i$, there exists $\delta_{i}>0$ that satisfies

$$
\delta_{i} \leq \frac{\varepsilon}{2 i+3}, \quad d_{Z}\left(z, z^{\prime}\right)<\delta_{i} \Longrightarrow\left|z-z^{\prime}\right|<\frac{1}{2(2 i+3) i}
$$

for all $z, z^{\prime} \in Z$. Choose $n_{i} \in \mathbb{N}$ such that $n_{i-1}<n_{i}$ and $\max \left(2^{-n_{i}}, \Delta_{n_{i}}\right)<\delta_{i} / 3$. For $i$ and for every $1 \leq m \leq n_{i}$, define $\varphi_{m}^{n_{i}}=\left\{\left(\pi_{m}^{n_{i}} \circ k_{n_{i}} \circ \xi\right)^{-1}\left(B\left(z, 2^{-n_{i}}\right)\right) \mid z \in Z_{m}\right\}$ (here, $B(z, r)$ stands for the open $d_{m}$-ball in $Z_{m}$ that is centered at $z$ with radius $r)$ and $\varphi_{0}^{n_{i}}=\left\{\left(k_{n_{i}} \circ \xi\right)^{-1}\left(W_{\alpha}\right)=\xi^{-1}\left(V_{\alpha}\right) \mid \alpha \in A\left(n_{i}\right)\right\}$. Write $\mathscr{G}_{0}=\varphi_{0}^{n_{i}} \cap \varphi_{0}^{n_{i-1}}$, and define $\mathscr{G}^{i}=\mathscr{G}_{0} \cap \bigcap_{m=1}^{n_{i-1}} \mathscr{G}_{m}^{n_{i-1}} \cap \bigcap_{m=1}^{n_{i}} \mathscr{G}_{m}^{n_{i}}$, an open cover of $Y$. Here, for open covers $\mathscr{A}_{1}, \ldots, \mathscr{A}_{p}$, we designate $\mathscr{A}_{1} \cap \cdots \cap \mathscr{A}_{p}=\left\{A_{1} \cap \cdots \cap A_{p} \mid A_{i} \in \mathscr{A}_{i}, i=\right.$ $1, \ldots, p\}$. Write $\mathscr{G}=\left\{G \cap Y_{i} \mid G \in \mathscr{G}^{i}\right.$ for some $\left.i\right\}$. Pick an open cover $\mathcal{U}$ that is a 
star refinement of $\mathscr{G}$. Using the fact $\operatorname{dim} Y_{i} \leq i$, find a partition of unity $\left\{\lambda_{U}\right\}_{U \in \mathscr{U}}$ such that $\operatorname{supp}\left(\lambda_{U}\right)=\lambda^{-1}((0,1]) \subset U$ and $\bigcap_{k=1}^{i+2} \operatorname{supp}\left(\lambda_{U_{k}}\right)=\varnothing$ if each $U_{k}$ is a subset of $Y_{i}$. For each $U \in \mathcal{U}$, choose the smallest $i \in \mathbb{N}$ with $U \subset Y_{i}$, and pick $z_{U} \in Z$ such that $\pi_{n_{i}}\left(z_{U}\right) \in k_{n_{i}} \circ \xi(U)$. Define $\eta(y)=\sum_{U \in \mathcal{U}} \lambda_{U}(y) z_{U}, y \in Y$.

Fix $y \in Y$ and write $A(y)=\left\{U \mid y \in \operatorname{supp}\left(\lambda_{U}\right)\right\}$. Suppose $i \in \mathbb{N}$ is the first index such that $y \in Y_{i}$. It may happen that $y \in Y_{i+1}$. Consequently, the cardinality of $A(y)$ is at most $2 i+3$. It also follows that $U(y)=\bigcup\{U \mid U \in A(y)\} \subset Y_{i} \cup$ $Y_{i+1}$. We conclude that for each $l=n_{i}$ or $n_{i+1}$, there exists $\alpha(l) \in A(l)$ such that $U(y) \subset\left(k_{l} \circ \xi\right)^{-1}\left(W_{\alpha(l)}\right)$. On the other hand, for each $U \in A(y)$ and for a certain such $l$, we have $\pi_{l}\left(z_{U}\right) \in k_{l} \circ \xi(U)$; so, we have $U \subset\left(k_{l} \circ \xi\right)^{-1}\left(W_{\alpha}\right)=\xi^{-1}\left(V_{\alpha}\right)$ and $\pi_{l}\left(z_{U}\right) \in k_{l} \circ \xi(U) \subset W_{\alpha}$ for $\alpha \in A(l)$, where $l=n_{i}$ or $l=n_{i+1}$. It shows that $\xi(y) \in V_{\alpha}$ and, by (2) of Lemma 3.1, that $d\left(\varphi\left(z_{U}\right), \bar{V}_{\alpha}\right) \leq 2 \Delta_{l}$; hence, $\| \varphi\left(z_{U}\right)-$ $\xi(y) \| \leq 3 \Delta_{l}<\max \left(\delta_{i}, \delta_{i+1}\right)$ because $l$ is either $n_{i}$ or $n_{i+1}$. We can estimate

$$
\begin{aligned}
\|\hat{\varphi}(\eta(y))-\xi(y)\| & =\left\|\sum_{U \in A(y)} \lambda_{U}(y)\left(\varphi\left(z_{U}\right)-\xi(y)\right)\right\| \\
& \leq(2 i+3)\left\|\varphi\left(z_{U}\right)-\xi(y)\right\| \\
& \leq(2 i+3) \max \left(\delta_{i}, \delta_{i+1}\right) \\
& \leq \varepsilon .
\end{aligned}
$$

The last inequality follows from the first part of (3.1). This shows (i).

To show (ii), we first partition the family $A(y)$ into $A^{1}(y)$ and $A^{2}(y) ; U \in$ $A^{j}(y)$ if and only if $i+j-1$ is the first index so that $U \subset Y_{i+j-1}, j=1,2$. As previously, for each $l=n_{i}$ or $n_{i+1}$ and $m \leq l$, there exists $z_{(l, m)} \in Z_{m}$ such that

$$
\begin{aligned}
U(y) \subset & \left(\pi_{m}^{l} \circ k_{l} \circ \xi\right)^{-1}\left(B\left(z_{(l, m)}, 2^{-l}\right)\right) \\
& =\left(k_{l} \circ \xi\right)^{-1}\left(\left(\pi_{m}^{n_{i}}\right)^{-1}\left(B\left(z_{(l, m)}, 2^{-l}\right)\right)\right) .
\end{aligned}
$$

Hence, if $\pi_{l}\left(z_{U}\right) \in k_{l} \circ \xi(U)$, then $\pi_{m}\left(z_{U}\right) \in B\left(z_{(l, m)}, 2^{-l}\right)$. It follows that, given $m$ with $1 \leq m \leq n_{i}, \pi_{m}\left(z_{U}\right) \in B\left(z_{\left(n_{i}, m\right)}, 2^{-n_{i}}\right)$ for all $U \in A^{1}(y)$; so, $\operatorname{diam}_{d_{m}}\left\{\pi_{m}\left(z_{U}\right) \mid\right.$ $\left.U \in A^{1}(y)\right\} \leq 2^{-n_{i}}$. Since

$$
\begin{aligned}
\operatorname{diam}_{d_{Z}}(S) & \leq \sum_{m=1}^{\infty} 2^{-m} \operatorname{diam}_{d_{m}}\left(\pi_{m}(S)\right) \\
& \leq\left(\sum_{m=1}^{n_{i}} 2^{-m} \operatorname{diam}(S)\right)+2^{-n_{i}} \quad \text { for every } S \subset Z,
\end{aligned}
$$

we conclude that $\operatorname{diam}_{d_{Z}}\left\{z_{U} \mid U \in A^{1}(y)\right\} \leq 2^{-n_{i}}+2^{-n_{i}} \leq \delta_{i}$. In a similar way, we show that $\operatorname{diam}_{d_{Z}}\left\{z_{U} \mid U \in A^{2}(y)\right\} \leq 2^{-n_{i+1}}+2^{-n_{i+1}} \leq \delta_{i+1}$. Hence, by the second part of (3.1),

$$
\left|z_{U_{1}}-z_{U_{1}^{\prime}}\right|<\frac{1}{2(2 i+3) i}, \quad\left|z_{U_{2}}-z_{U_{2}^{\prime}}\right|<\frac{1}{2(2 i+5)(i+1)}
$$


for all $z_{U_{1}}, z_{U_{1}^{\prime}} \in A^{1}(y)$ and all $z_{U_{2}}, z_{U_{2}^{\prime}} \in A^{2}(y)$. Fix $z_{U_{j}}$, where $U_{j} \in A^{j}(y)$, and let $t_{j}=\sum\left\{\lambda_{U}(y) \mid U \in A^{j}(y)\right\}, j=1,2$. From (3.5), we obtain

$$
\begin{aligned}
\left|\eta(y)-\left(t_{1} z_{U_{1}}+t_{2} z_{U_{2}}\right)\right| & =\left|\sum_{U \in A(y)} \lambda_{U}(y)\left(z_{U}-z_{U_{1}}\right)+\sum_{U \in A^{2}(y)} \lambda_{U}(y)\left(z_{U}-z_{U_{2}}\right)\right| \\
& \leq(2 i+3)\left(\frac{1}{2(2 i+3) i}+\frac{1}{2(2 i+5)(i+1)}\right)<\frac{1}{i} .
\end{aligned}
$$

Now, if $\left\{\eta\left(y_{k}\right)\right\}$ is a sequence in $\operatorname{conv}(Z)$, then either $\left\{y_{k}\right\}$ has a subsequence contained in some $Y_{i}$ (this subsequence, in turn, contains a convergent subsequence because $\eta\left(\bar{Y}_{i}\right)$ is compact), or else it contains a subsequence $\left\{y_{i(n)}\right\}$ such that $y_{i(n)} \in Y_{i(n)}$ for all $n$. In the latter instance, from the above estimate, we have $\left|\eta\left(y_{i(n)}\right)-\left(t_{n}^{1} z_{n}^{1}+t_{n}^{2} z_{n}^{1}\right)\right| \leq 1 / i(n)$ for some $t_{n}^{1}, t_{n}^{2} \geq 0$ with $t_{n}^{1}+t_{n}^{2}=1$ and some $z_{n}^{1}, z_{n}^{2} \in Z$. By the compactness of $Z$, it now easily follows that $\left\{\eta\left(y_{i(n)}\right)\right\}$ contains a subsequence that converges to $\operatorname{conv}_{2}(Z)$. Since $\operatorname{conv}_{2}(Z)$ is compact, (ii) is shown.

In what follows, we present a general overview of the argument that justifies Lemma 3.1. More precisely, we give a sketch of the proof of Lemma 3.1 assuming that $\left\{V_{\alpha}\right\}$ and the inverse sequence $\left(Z_{n}, \pi_{n}^{n+1}\right)$ satisfy conditions (3.7), (3.8), and (3.9). We will not comment on the construction of the sequence $\left(Z_{n}, \pi_{n}^{n+1}\right)$. This will be done in Section 4.

Remark 3.2. The continuity of $\varphi$ is easily obtained from condition (2) of Lemma 3.1 as follows. For every $n,\left\{\pi_{n}^{-1}\left(W_{\alpha}\right) \mid \alpha \in A(n)\right\}$ is an open cover of $Z$. If $z, z^{\prime} \in \pi_{n}^{-1}\left(W_{\alpha}\right)$, then $d\left(\varphi(z), \varphi\left(z^{\prime}\right)\right) \leq d\left(\varphi(z), \bar{V}_{\alpha}\right)+\operatorname{diam}\left(\bar{V}_{\alpha}\right)+d\left(\varphi\left(z^{\prime}\right), \bar{V}_{\alpha}\right) \leq$ $5 \Delta_{n} \rightarrow 0$.

Now, we first define $\varphi$ in case (3.7) holds.

Remark 3.3. Fix $z \in Z$ and let $A(z)=\left\{\alpha \in A(n) \mid \pi_{n}(z) \in W_{\alpha}\right\}$. Define $F_{n}(z)=$ $\bigcap\left\{\bar{V}_{\alpha} \mid \alpha \in A(z)\right\}$. Assume

$$
d_{H}\left(F_{n}(z), F_{m}(z)\right) \leq 2 \Delta_{n}
$$

for $1 \leq n \leq m$ (here, $d_{H}$ stands for the Hausdorff metric induced by $d$ ). Let $\varphi(z)=x$, where $\{x\}=\lim _{d_{H}} F_{n}(z)$. Note that (3.7), together with condition (3) of Lemma 3.1, implies that the sequence $\left\{F_{n}(z)\right\}$ converges to a singleton in the hyperspace of $X$; hence, $\varphi$ is well defined. On the other hand, $F_{n}(z) \subset \bar{V}_{\alpha}$ for all $\alpha \in A(z)$. Applying (3.7) again, we easily obtain (2) of Lemma 3.1.

Next, we indicate how the countable dimensionality of $Z$ can be achieved (here, we can compare this argument with that of Zarichny [30]). We stress that the countable dimensionality of $Z$ is essential for deducing Corollary 2.6 from Proposition 2.4. 
Remark 3.4. Suppose that each $Z_{n}$ is a tower of compacta $Z_{n}^{k}$ (in Section 4, denoted by $\left.M_{n}^{k}\right), 0 \leq k<n$, such that

$$
\operatorname{dim}\left(Z_{n}^{k}\right) \leq k, \quad\left(\pi_{n}^{n+1}\right)^{-1}\left(Z_{n}^{k}\right) \subset Z_{n+1}^{k}
$$

for every $0 \leq k<n$. Furthermore, suppose $Z_{n} \backslash Z_{n}^{n-1}=\bigcup \boldsymbol{U}_{n}$ for a certain family $u_{n}=\left\{U_{\beta} \mid \beta \in B(n)\right\}$ that consists of finite, pairwise disjoint open subsets of $Z_{n}$ with

$$
\operatorname{diam}_{d_{m}}\left(\pi_{m}^{n}\left(U_{\beta}\right)\right) \leq 2^{-n+1}
$$

for every $0 \leq m<n$ and $\beta \in B(n)$. Then, we have $Z=P_{0} \cup \bigcup_{n=1}^{\infty} P_{n}$, where $P_{0}=$ $\bigcap_{n=1}^{\infty} \pi_{n}^{-1}\left(\cup \mathcal{U}_{n}\right)$ and $P_{n}=\pi_{n}^{-1}\left(Z_{n}^{n-1}\right)$. Since condition (3.9) yields $\operatorname{diam}_{d_{Z}}\left(\pi_{n}^{-1}\right.$ $\left.\left(U_{\beta} B\right)\right)<2^{-n+2}$ for every $\beta \in B(n), n \in \mathbb{N}$, it follows that for every $n, P_{0}$ can be covered by the finite family $\left\{\pi_{n}^{-1}\left(U_{\beta}\right) \mid \beta \in B(n)\right\}$ which consists of open pairwise disjoint sets of diameter $<2^{-n+2}$; hence, $\operatorname{dim}\left(P_{0}\right) \leq 0$. On the other hand, it can be easily checked that $P_{n}=\pi_{n}^{-1}\left(Z_{n}^{n-1}\right)=\underline{\lim }\left(\pi_{m}\left(P_{n}\right), \pi_{m}^{k} \mid \pi_{k}\left(P_{n}\right)\right)$. From (3.8), we infer that $\pi_{m}\left(P_{n}\right) \subset Z_{m}^{n-1}$ (and consequently, $\operatorname{dim}\left(\pi_{m}\left(P_{n}\right)\right) \leq n-1$ because $\left.\operatorname{dim}\left(Z_{m}^{n-1}\right) \leq n-1\right)$ for all $m \geq n$. This yields $\operatorname{dim}\left(P_{n}\right) \leq n-1$.

In the case that $X$ is merely a uniformly contractible compactum, the proof of Proposition 2.4 requires the following adjustments (that were initiated at the end of Section 2). Formally, the definition of $\eta$ is the same, but to guarantee Proposition 2.4(i) and (ii), we must modify the choice of $\delta_{i}$ made in condition (3.1). Assuming $\delta_{i+1} \leq \delta_{i}$, it suffices to have $d\left(\mu_{2 i+3}\left(x_{1}, \ldots, x_{2 i+3} ; t_{1}, \ldots, t_{2 i+3}\right), x\right) \leq$ $\varepsilon$ for $d\left(x_{i}, x\right)<\delta_{i}$ and $i=1, \ldots, 2 i+3$, and $\rho\left(t_{1} z_{1}+\cdots+t_{2 i+5} z_{2 i+5}+t_{1}^{\prime} z_{1}^{\prime}+\cdots+\right.$ $\left.t_{2 i+5}^{\prime} z_{2 i+5}^{\prime}, t z+t^{\prime} z^{\prime}\right)<1 / i$ for $d_{Z}\left(z_{i}, z\right)<\delta_{i}$ and $d_{Z}\left(z_{i}^{\prime}, z^{\prime}\right)<\delta_{i}, i=1, \ldots, 2 i+5$, where $\left(t_{1}, \ldots, t_{2 i+5}, t_{1}^{\prime}, \ldots, t_{2 i+5}^{\prime}\right) \in s_{4 i+10}$ and $t=\sum_{i=1}^{2 i+5} t_{i}, t^{\prime}=\sum_{i=1}^{2 i+5} t_{i}^{\prime}$.

\section{The proof of Lemma 3.1}

We begin with a statement of the three main points of Cauty's original construction of $\varphi: Z \rightarrow X$. Having done this, we show how Lemma 3.1 can be deduced. The detailed Cauty's construction is performed in Sections 4.1 and 4.2.

Step 1. With the metric compactum $X=(X, d)$, we associate an inverse sequence $\left(K_{n}, q_{n}\right)$ of finite simplicial complexes $K_{n}, \operatorname{dim}\left(K_{n}\right) \leq n$, which are nerves of certain open finite covers $\mathscr{V}_{n}=\left\{V_{\alpha} \mid \alpha \in A_{n}\right\}$ of $X$, which is indexed in such a way that $\alpha \neq \alpha^{\prime}$ implies $V_{\alpha} \neq V_{\alpha^{\prime}}$. Writing $K_{n}=\operatorname{nerv}\left(\mathscr{V}_{n}\right)$, we identify elements of $A_{n}$ with the vertices of $K_{n}$. Later, the set of the vertices of $K$ is denoted by $\operatorname{Vert}(K)$; for a vertex $\alpha \in \operatorname{Vert}(K)$, st $(\alpha, K)$ stands for the open star of $\alpha$ in $|K|$, the body of $K$. We further assume that

(K1) $\mathscr{V}_{n+1} \prec \mathscr{V}_{n}$ (i.e., $\mathscr{V}_{n+1}$ is inscribed in $\mathscr{V}_{n}$ ) and the simplicial map $q_{n}$ : $K_{n+1} \rightarrow K_{n}$ is onto, which is a consequence of $(\mathrm{K} 2)$; 
(K2) each canonical map

$$
\psi_{n}: X \longrightarrow\left|K_{n}\right|
$$

is onto; recall that $\psi_{n}^{-1}\left(\operatorname{st}\left(\alpha, K_{n}\right)\right)=V_{\alpha}, \alpha \in A ;$

(K3) $\operatorname{dim}\left(K_{n}\right) \leq n$;

(K4) $\Delta_{n}=\sup \left\{\operatorname{diam}_{d}\left(V_{\alpha}\right) \mid \alpha \in A_{n}\right\} \rightarrow 0$ as $n \rightarrow \infty$.

We are not going to elaborate on justifying the assertion of this step which, in a sense, belongs to the topological folklore (see $[23,24,27])$. Note that though we could have additionally arranged $X=\lim \left(K_{n}, q_{n}\right)$, we do not request that. Our objective is just the opposite: to "modify" this inverse sequence in order to get a countable-dimensional compactum as its limit.

Step 2. The space $Z$ is constructed as the inverse limit of the sequence $\left(Z_{n}, \pi_{n}^{n+1}\right)$ of finite-dimensional compacta $Z_{n}$ so that $\left|K_{n}\right| \subset Z_{n}$ and $\left|K_{n}\right|$ is a retract of $Z_{n}$; that is, there exists a retraction $\rho_{n}: Z_{n} \rightarrow\left|K_{n}\right|$. Moreover, we have

$$
\pi_{n}^{n+1}\left(\left|K_{n+1}\right|\right) \supset\left|K_{n}\right|
$$

for every $n$. The compactum $Z_{n}$ is built in two stages; first, we enlarge $K_{n}$ to yet another finite complex $M_{n}=M\left(K_{n}^{\left(k_{n}\right)}, n\right)$, and then the body $\left|M_{n}\right|$ is enlarged to the compactum $Z_{n}$. Write $\pi_{n}: Z=\longleftarrow\left(Z_{n}, \pi_{n}^{n+1}\right) \rightarrow Z_{n}$ for the natural projection. Condition (4.2) guarantees that $\pi_{n} \overleftarrow{(Z)} \supset\left|K_{n}\right|$ for every $n$.

Step 3. For a point $z \in Z$, we define a subcompactum $F_{n}(z) \subset X$ as follows. To $z$ we assign the smallest simplex $s_{n}(z) \in K_{n}$ containing $\rho_{n} \circ \pi_{n}(z)$. Then $F_{n}(z)=$ $\bigcap\left\{\bar{V}_{\alpha} \mid V_{\alpha} \in \mathscr{V}_{n}\right.$ is a vertex of $\left.s_{n}(z)\right\}$. For every $z$, the sequence of compacta $\left(F_{n}(z)\right)$ is convergent to a singleton; we set

$$
\varphi(z)=\lim _{n} F_{n}(z)
$$

Lemma 4.1, a counterpart of Lemma 3.1, contains a fact that is detached from Cauty's original work related to the construction of $\varphi: Z \rightarrow X$. Following the statement, we note that actually Lemma 4.1 implies Lemma 3.1; this shows that the original construction of Cauty yields Lemma 3.1. The proof of Lemma 4.1 will be given in Sections 4.1 and 4.2.

Lemma 4.1. Steps 1, 2, and 3 can be performed in order, for $Z=\underset{\lim }{L}\left(Z_{n}, \pi_{n}^{n+1}\right)$, to be a countable-dimensional compactum and, for the map $\varphi: Z \rightarrow \overleftarrow{X}$, to satisfy the following condition:

$$
d\left(\varphi(z), \bar{V}_{\alpha}\right) \leq 2 \Delta_{n}
$$

for all $z \in Z$ and all $\alpha \in A_{n}$, such that $\rho_{n} \circ \pi_{n}(z) \in \operatorname{st}\left(\alpha, K_{n}\right)$. 
Proof of Lemma 3.1. Let $K_{n},\left\{V_{\alpha} \mid \alpha \in A_{n}\right\}, \rho_{n}$, and $\psi_{n}$ be that of Lemma 4.1. Designate $W_{\alpha}=\rho_{n}^{-1}\left(\operatorname{st}\left(\alpha, V_{\alpha}\right)\right), A(n)=A_{n}$, and $k_{n}=\psi_{n}$. We see that $\psi_{n}^{-1}\left(W_{\alpha}\right)=$ $\psi_{n}^{-1}\left(\operatorname{st}\left(\alpha, V_{\alpha}\right)\right)=V_{\alpha}$ for $\rho_{n} \circ \pi_{n}(z) \in \operatorname{st}\left(\alpha, K_{n}\right)$ if and only if $\pi_{n}(z) \in \rho_{n}^{-1}\left(\operatorname{st}\left(\alpha, K_{n}\right)\right)=$ $W_{\alpha}$. Now, conditions (1), (2), and (3) of Lemma 3.1 follow from (K4) and (4.4a) of Lemma 4.1.

Note 4.2. In deriving Proposition 2.4 from Lemma 4.1, the full power of (4.4a) is needed only to show the continuity of $\varphi$. (As in Remark 3.2, for every $n,\left\{\left(\rho_{n}\right.\right.$ 。 $\left.\left.\pi_{n}\right)^{-1}\left(\operatorname{st}\left(\alpha, K_{n}\right)\right) \mid \alpha \in A_{n}\right\}$ is an open cover of $Z$. Now, if $z, z^{\prime} \in\left(\rho_{n} \circ \pi_{n}\right)^{-1}(\operatorname{st}(\alpha$, $\left.\left.K_{n}\right)\right)$, then $d\left(\varphi(z), \varphi\left(z^{\prime}\right)\right) \leq d\left(\varphi(z), \bar{V}_{\alpha}\right)+\operatorname{diam}\left(\bar{V}_{\alpha}\right)+d\left(\varphi\left(z^{\prime}\right), \bar{V}_{\alpha}\right) \leq 5 \Delta_{n}$. Since $\lim \Delta_{n}=0$, the continuity of $\varphi$ follows.) The remaining part of the proof of Proposition 2.4 employs the following weaker version of condition (4.4a):

$$
d\left(\varphi(z), \bar{V}_{\alpha}\right) \leq 2 \Delta_{n}
$$

for all $z \in Z$ and all $\alpha \in A_{n}$, such that $\pi_{n}(z) \in\left|K_{n}\right|$ and $\pi_{n}(z) \in \operatorname{st}\left(\alpha, K_{n}\right)$.

4.1. The compactum $Z$. In this subsection, which is split in a few units, we gradually provide details related to the statement of Step 2 .

4.1.1. Construction and properties of $M(K, n)$. Let $K$, a finite complex, and $n=$ $0,1,2, \ldots$ be given so that $\operatorname{dim}(K) \leq n$. For a simplex $\sigma \in K, b_{\sigma}$ denotes the barycenter of $\sigma$. By $K^{(1)}$ we denote the first barycentric subdivision of $K\left(K^{(l)}\right.$ denotes the $l$ th barycentric subdivision of $K) ; K^{(1)}$ consists of simplices $\left(b_{\sigma_{0}}, \ldots, b_{\sigma_{k}}\right)$ where $\sigma_{0} \leq \cdots \leq \sigma_{k}$ (i.e., $\sigma_{0} \subseteq \cdots \subseteq \sigma_{k}$ ), $\sigma_{j} \in K$. Mimicking this pattern, we construct a finite simplex $M(K, n)$ associated with $K$ and $n$. We let

$$
\operatorname{Vert}(M(K, n))=\left\{v=\left(b_{\sigma}, m\right) \mid \sigma \in K, m \in\{\operatorname{dim}(\sigma), 1+\operatorname{dim}(\sigma), \ldots, n\}\right\}
$$

A finite set of vertices $\left\{v_{0}=\left(b_{\sigma_{0}}, m_{0}\right), \ldots, v_{k}=\left(b_{\sigma_{k}}, m_{k}\right)\right\}$ is a simplex of $M(K, n)$ if

$$
\sigma_{o} \leq \cdots \leq \sigma_{k}, \quad m_{0}<\cdots<m_{k}
$$

The retraction $r=r(K, n): M(K, n) \rightarrow K^{(1)}$. Define $r\left(\left(b_{\sigma}, m\right)\right)=b_{\sigma}$ to obtain a simplicial map of $M(K, n)$ onto $K^{(1)}$. Identifying $K^{(1)}$ with a subcomplex $M(K, n)$ generated by the vertices $\left(b_{\sigma}, \operatorname{dim}(\sigma)\right)$, we see that such $r=r(K, n)$ is a simplicial retraction of $M(K, n)$ onto $K^{(1)}$.

The subcomplex $M^{k}(K, n)$. For $0 \leq k \leq n$, define $M^{k}(K, n)$ as a subcomplex of $M(K, n)$ generated by the vertices $\left(b_{\sigma}, m\right)$ with $m \leq k$. Notice that $K^{(1)} \subset$ $M^{\operatorname{dim}(\sigma)}(K, n)$ and $M^{n}(K, n)=M(K, n)$. 
The map $v(x)=v(K, n)(x)$. For $x \in|M(K, n)|$, define

$$
v(x)=\sum\left\{t_{v}(x) \mid v=\left(b_{\sigma}, m\right) \in \operatorname{Vert}(M(K, n)) \text { with } m>\operatorname{dim}(\sigma)\right\}
$$

where $t_{v}(x)$ stands for the $v$ th barycentric coordinate of $x$. Note that $v|| K \mid=0$.

The following lemma shows that $K^{(1)}$ is a deformation retract of $M(K, n)$; furthermore, there exists a deformation (whose other technical properties are listed below) that preserves the fibres of $r$.

Lemma 4.3 (cf. [5, Lemma 4]). There exists a homotopy $H:|M(K, n)| \rightarrow|M(K, n)|$ satisfying

(i) $H(x, 0)=x$ for every $x \in|M(K, n)|$ and $H(x, t)=x$ for every $x \in|K|$ and $0 \leq t \leq 1$,

(ii) $H(x, 1)=r(x)$ for every $x \in|M(K, n)|$,

(iii) (for all $(x, t) \in|M(K, n)| \times[0,1])[r(H(x, t))=r(x)]$,

(iv) (for all $0 \leq k \leq n)\left[x \in\left|M^{k}(K, n)\right| \Rightarrow H(x, t) \in\left|M^{k}(K, n)\right|\right]$,

(v) (for all $0 \leq k \leq n)\left[x \in\left|M^{k}(K, n)\right| \Rightarrow H(x, t)=H(x, k / n)=H(x, 1)\right.$ if $k / n \leq t \leq 1]$

(vi) (for all $x \in|M(K, n)|)[t \rightarrow v(H(x, t))$ is decreasing],

(vii) (for all $x \in|M(K, n)|$ ) (for all $t \in[0,1]$ ) (for all $v=\left(b_{\tau}, m\right)$ ) $\left[t_{v}(H(x, t)) \neq\right.$ $0 \Rightarrow m=\operatorname{dim}(\tau)$ or $m \leq k]$, where $k=\max \left\{m \mid v=\left(b_{\sigma}, m\right)\right.$ with $m>$ $\operatorname{dim}(\sigma)$ and $\left.t_{v}(x) \neq 0\right\}$

(viii) (for all $x \in|M(K, n)|$ ) (for all $v=\left(b_{\sigma}, m\right) \in \operatorname{Vert}(M(K, n)$ ) with $m>0$ ) $\left[t_{v}(x)>0\right.$ and $\operatorname{dim}(\sigma)<m \Rightarrow[(m-1) / n, m / n] \ni t \rightarrow t_{v}(H(x, t))$ linearly decreases to zero]; in the remaining case (i.e., whenever $t_{v}(x)=0$ or $\operatorname{dim}(\sigma)$ $=m)$ for every $(m-1) / n \leq t \leq m / n, t_{v}(H(x, t))$ is constant.

Proof. Letting $H(x, 0)=x$, we first define $H$ over the interval $0 \leq t \leq 1 / n$. Namely, for $v=\left(b_{\sigma}, m\right) \in \operatorname{Vert}(M(K, n))$, we let $H(v, t)=v$ if $m=\operatorname{dim}(\sigma)$, and $H(v, t)=$ $(1-n t)\left(b_{\sigma}, m\right)+n t\left(b_{\sigma}, \operatorname{dim}(\sigma)\right)$ if $m=1$. Extend this homotopy affinely with respect to $t$ over all simplices of $M(K, n)$, and notice that $H(x, 1 / n)$ belongs to a subcomplex $L_{1}$ of $M(K, n)$ that is generated by the vertices $v=\left(b_{\sigma}, m\right)$ for which either $m=\operatorname{dim}(\sigma)$ or $m \geq 2$.

For $1 / n \leq t \leq 2 / n, H(x, t)$ will be of the form $G(H(x, 1 / n), t)$, where $G$ is a certain homotopy defined on $L_{1} \times[1 / n, 2 / n]$. Namely, for $v=\left(b_{\sigma}, m\right) \in \operatorname{Vert}\left(L_{1}\right)$, let $G(v, t)=v$ if $m \neq 2$, and $G(v, t)=(2-n t)\left(b_{\sigma}, m\right)+(n t-1)\left(b_{\sigma}, \operatorname{dim}(\sigma)\right)$, otherwise. To extend $G$ over the simplices of $L_{1}$, it is enough to consider a simplex $\left\{\left(b_{\sigma_{0}}, m_{0}\right), \ldots,\left(b_{\sigma_{k}}, m_{k}\right)\right\} \in L_{1}\left(m_{0}<\cdots<m_{k}\right)$ such that, for some $j \geq 1$, $\operatorname{dim}\left(\sigma_{j}\right)<m_{j}=2$. Then $m_{j-1}<2$, and hence $m_{j-1}=\operatorname{dim} \sigma_{j-1} \leq \operatorname{dim} \sigma_{j}$. It follows that $\left\{\left(b_{\sigma_{0}}, m_{0}\right), \ldots,\left(b_{\sigma_{j-1}}, m_{j-1}\right),\left(b_{\sigma_{j}}, \operatorname{dim} \sigma_{j}\right),\left(b_{\sigma_{j}}, m_{j}\right), \ldots,\left(b_{\sigma_{k}}, m_{k}\right)\right\}$ is a simplex of $L_{1}$ (we obviously have $\left(b_{\sigma_{j-1}}, m_{j-1}\right)=\left(b_{\sigma_{j}}, \operatorname{dim} \sigma_{j}\right)$ if $\operatorname{dim} \sigma_{j-1}=\operatorname{dim} \sigma_{j}$ ). This allows us to extend $G$ affinely with respect to $t$ over $\left|L_{1}\right|$ and conclude that $H(x, 2 / n)$ will be a point of a subcomplex $L_{2}$ generated by the vertices $v=\left(b_{\sigma}, m\right)$ for which either $m=\operatorname{dim}(\sigma)$ or $m \geq 3$. 
After finitely many steps, we finally define $H$ over the interval $[(n-1) / n, 1]$ such that $H(x, 1) \in L_{n}=K^{(1)}$. Since, for every vertex $v$, we have $r \circ H(v, t)=$ $r(v)$, it follows that $r \circ H(x, t)=r(x)$ for every $x$; in particular, $H(x, 1)=r(x)$. Properties (iv), (v), (vi), (vii), and (viii) also follow.

Corollary 4.4. The following holds:

(i) (for all $\sigma \in K)\left[D_{\sigma}=r^{-1}(|\sigma|)\right.$ is contractible $]$,

(ii) the family $\left\{D_{\sigma} \backslash\left|M^{n-1}(K, n)\right| \mid \sigma \in K\right\}$ is finite and consists of open, pairwise disjoint sets of $|M(K, n)|$.

Proof. Item (i) follows from Lemma 4.3(iii). To prove item (ii), observe that for each $\sigma \in K, r^{-1}(\sigma) \backslash\left|M^{n-1}(K, n)\right|=\operatorname{st}(v, M(K, n))$, where $\operatorname{st}(v, M(K, n))$ is the open star of the vertex $v=\left(b_{\sigma}, n\right) \in M(K, n)$. No such stars have a point in common since there is no simplex in $M(K, n)$ with two vertices of this kind (i.e., for any other vertex $\left(b_{\tau}, m\right)$, we must have $\left.m<n\right)$.

Corollary 4.5 ([5, Lemma 5]). For every $\varepsilon>0$, there exists a map $h_{\varepsilon}:|M(K, n)| \rightarrow$ $|M(K, n)|$ such that

(i) $h_{\varepsilon}|| K \mid=\mathrm{id}_{|K|}$,

(ii) (for all $x \in|M(K, n)|)\left[r\left(h_{\varepsilon}(x)\right)=r(x)\right]$,

(iii) (for all $0 \leq k \leq n)\left[h_{\varepsilon}^{-1}\left(\left|M^{k}(K, n)\right|\right)=\left|M^{k}(K, n)\right|\right]$,

(iv) (for all $x \in|M(K, n)|$ ) $\left[v\left(h_{\varepsilon}(x)\right) \leq \varepsilon\right]$.

Proof. Let $H$ be a homotopy of Lemma 4.3. Define $\alpha(x)=\sup \{t \in[0,1] \mid$ $v(H(x, t)) \geq \varepsilon\}($ let $\sup \varnothing=0)$ and $\beta(x)=\inf \{t \in[0,1] \mid v(H(x, t))=0\} ; \alpha$ is upper semicontinuous and $\beta$ is lower semicontinuous. Denote by $G$ the complement of $\beta^{-1}(0)$; $G$ is an open set. By Lemma 4.3(vi), for all $x \in G, \alpha(x)<\beta(x)$. There exists a continuous function $\gamma: G \rightarrow[0,1]$ such that $\alpha(x)<\gamma(x)<\beta(x)$, $x \in G$. Extend $\gamma$ to a (necessarily) continuous function over the whole $|M(K, n)|$ by letting $\gamma(x)=0$ off $G$. Finally, let $h_{\varepsilon}(x)=H(x, \gamma(x))$.

Properties (i), (ii), and (iv) follow from those (i) and (iii) of Lemma 4.3 and the definition of $\alpha$. Taking into account Lemma 4.3(iv), property (iii) reduces to $h_{\varepsilon}^{-1}\left(\left|M^{k}(K, n)\right|\right) \subset\left|M^{k}(K, n)\right|$ for $0 \leq k \leq n$. To show this, let $x \notin\left|M^{k}(K, n)\right|$, and additionally, $x \notin|K|$; the latter implies $\beta(x)>0$. Find the smallest simplex $\left\{\left(b_{\sigma_{0}}, m_{0}\right), \ldots,\left(b_{\sigma_{p}}, m_{p}\right)\right\}$ containing $x$. Then $m_{p}>k$ and, by Lemma $4.3(\mathrm{v})$, $H(x, t)=H\left(x, m_{p} / n\right) \in|K|$ for all $t \geq m_{p} / n$. Since $\gamma(x)<\beta(x) \leq m_{p} / n$, by an application of Lemma 4.3 (viii) to the vertex $v=\left(b_{\sigma_{p}}, m_{p}\right)$, we get $t_{v}(H(x, \gamma(x))) \neq$ 0 ; hence, $h_{\varepsilon}(x) \notin\left|M^{k}(K, n)\right|$.

For $l \geq 1$, consider the complex $M\left(K^{(l)}, n\right)$ associated with $K^{(l)}$, the $l$ th barycentric subdivision of $K$. Together with the map $r: M(K, n) \rightarrow K^{(1)}$, we consider $r_{1}=r\left(K^{(l)}, n\right): M\left(K^{(l)}, n\right) \rightarrow\left(K^{(l)}\right)^{(1)}=K^{(l+1)}$. In the next lemma, we list properties of an important map $\left|M\left(K^{(l)}, n\right)\right| \rightarrow|M(K, n)|$ that will be used in constructing $\pi_{n}^{n+1}$. 
Lemma $4.6\left(\left[5\right.\right.$, Lemma 6]). There exists $f:\left|M\left(K^{(l)}, n\right)\right| \rightarrow|M(K, n)|$ satisfying

(i) $f|| K \mid=\mathrm{id}_{|K|}$,

(ii) (for all $\left.x \in\left|M\left(K^{(l)}, n\right)\right|\right)\left[d\left(r(f(x)), r_{1}(x)\right)<\varepsilon\right]$,

(iii) (for all $0 \leq k \leq n)\left[f^{-1}\left(\left|M^{k}(K, n)\right|\right) \subset\left|M^{k}\left(K^{(l)}, n\right)\right|\right]$,

(iv) (for all $\left.\sigma \in K^{(l)}\right)\left[f\left(r_{1}^{-1}(|\sigma|)\right) \subset r^{-1}(|\sigma|)\right]$,

(v) (for all $\left.x \in\left|M\left(K^{(l)}, n\right)\right|\right)[v(f(x))<\varepsilon]$.

Proof. Notice that if $f$ satisfies (i), (ii), (iii), and (iv), then, letting $h_{\varepsilon}$ be that of Corollary 4.5, $h_{\varepsilon} \circ f$ satisfies all conditions (i), (ii), (iii), (iv), and (v). Furthermore, we claim that it is enough to consider the case of $l=1$. Suppose $f_{i}:\left|M\left(K^{(i)}, n\right)\right|=\left|M\left(\left(K^{(i-1)}\right)^{(1)}, n\right)\right| \rightarrow\left|M\left(K^{(i-1)}, n\right)\right|, 1 \leq i \leq l$, has been constructed in order to satisfy conditions (i), (ii), (iii), (iv), (v) (with a suitable $\varepsilon_{i}>0$ ), and (vi) below. Then the composition $f_{1} \circ \cdots \circ f_{l}$ can serve for $f$.

We use induction with respect to $\operatorname{dim}(K)$ to construct the requested $f:\left|M\left(K^{(1)}, n\right)\right| \rightarrow|M(K, n)|$; we impose the following extra condition on $f$ :

(vi) (for all $x \in\left|M^{k}\left(K^{(1)}, n\right)\right|$ ) (for all $\left.v=\left(b_{\sigma}, m\right) \in \operatorname{Vert}(M(K, n))\right)\left[t_{v}(f(x))\right.$ $\neq 0 \Rightarrow m=\operatorname{dim}(\sigma)$ or $m \leq k]$.

If $\operatorname{dim}(K)=0$, then $f=$ id will work. So, letting $\operatorname{dim}(K)=d>0$, we may assume that the assertion of Lemma 4.6 holds for all complexes of dimension less than or equal to $d-1$. Denote by $K_{d-1}$ the $(d-1)$ skeleton of $K$ and let $f^{\prime}$ : $\left|M\left(K_{d-1}^{(1)}, n\right)\right|=r_{1}^{-1}\left(\left|K_{d-1}\right|\right) \rightarrow\left|M\left(K_{d-1}, n\right)\right|=r^{-1}\left(\left|K_{d-1}\right|\right)$ be a map that satisfies (i), (ii), (iii), (iv), and (vi) with $\varepsilon>0$. Our goal is to extend $f^{\prime}$ to a required map $f$ step by step over all simplices of dimension $d$.

Fix $s \in K, \operatorname{dim}(s)=d$, and let $b_{s}$ be its barycenter. Write $V=\left\{v \in \operatorname{Vert}\left(M\left(K^{(1)}\right.\right.\right.$, $\left.n)) \mid r_{1}(v) \in s^{(2)}\right\}$ and let

$$
S=\left\{v \in V \mid r_{1}(v) \in \overline{\operatorname{st}}\left(b_{s}, K^{(2)}\right)\right\}, \quad T=\left\{v \in V \mid r_{1}(v) \in(\partial s)^{(2)}\right\}
$$

we have $V=S \cup T$. For $x \in r_{1}^{-1}(|s|)$, define

$$
\alpha(x)=\sum_{v \in S} t_{v}(x), \quad \beta(x)=\sum_{v \in T} t_{v}(x)
$$

clearly, $\alpha(x)+\beta(x)=1$. First, we define auxiliary maps $\xi: r_{1}^{-1}($ int $|s|) \rightarrow r^{-1}\left(b_{s}\right)$ by

$$
\xi(x)=\sum_{v=\left(b_{\sigma}, m\right) \in S} \frac{t_{v}(x)}{\alpha(x)}\left(b_{s}, \max (m, d)\right),
$$

and $\eta: r_{1}^{-1}\left(|s| \backslash\left|\overline{\operatorname{st}}\left(b_{s}, K^{(2)}\right)\right|\right) \rightarrow r_{1}^{-1}(|\partial s|)$ by

$$
\eta(x)=\sum_{v \in T} \frac{t_{v}(x)}{\beta(x)} v .
$$


We let $\pi=\rho \circ r_{1}: r_{1}^{-1}\left(|s| \backslash\left\{b_{s}\right\}\right) \rightarrow|\partial s|$, where $\rho:|s| \backslash\left\{b_{s}\right\} \rightarrow|\partial s|$ is the radial projection. For $x \in r_{1}^{-1}\left(|s| \backslash\left\{b_{s}\right\}\right)$, the equation

$$
r_{1}(x)=(1-\mu(x)) \pi(x)+\mu(x) b_{s}
$$

determines a continuous function $\mu: r_{1}^{-1}\left(|s| \backslash\left\{b_{s}\right\}\right) \rightarrow[0,1]$.

Choose open sets $U_{1}, U_{2} \subset r_{1}^{-1}(|s|) \backslash|s|$ such that $r_{1}^{-1}(|\partial s|) \backslash|s| \subset U_{1} \subset U_{2}$ and

$$
\bar{U}_{2} \subset r_{1}^{-1}\left(|s| \backslash\left|\overline{s t}\left(b_{s}, K^{(2)}\right)\right|\right), \quad \bar{U}_{2} \cap|s|=|\partial s|, \quad \bar{U}_{1} \subset U_{2} \cup|\partial s| ;
$$

here, the closure is taken in $r_{1}^{-1}(|s|)$. Finally, we employ two steering functions $\gamma, \delta: r_{1}^{-1}(|s|) \backslash|s| \rightarrow[0,1]$ such that

$$
\begin{gathered}
\gamma\left|r_{1}^{-1}(|\partial s|) \backslash\right| s \mid=0, \quad \gamma(x)=1 \quad \text { if } x \notin U_{1}, \\
\delta \mid U_{1}=0, \quad \delta(x)=1 \quad \text { if } x \notin U_{2} .
\end{gathered}
$$

Letting $H$ be a homotopy of Lemma 4.3, we define a required map $f \mid r_{1}^{-1}(|s|)$ : $r_{1}^{-1}(|s|) \rightarrow r^{-1}(|s|)$ by

$$
f(x)=\left\{\begin{array}{lr}
f^{\prime}(x) \quad \text { if } x \in r_{1}^{-1}(|\partial s|), \\
(1-\mu(x)) H\left(f^{\prime} \circ \eta(x), \gamma(x)\right)+\mu(x) \xi(x) \quad & \text { if } x \in \bar{U}_{1} \backslash r_{1}^{-1}(|\partial s|), \\
(1-\mu(x))\left[(1-\delta(x)) r \circ f^{\prime} \circ \eta(x)+\delta(x) \pi(x)\right]+\mu(x) \xi(x) & \text { if } x \in \bar{U}_{2} \backslash\left(U_{1} \cup|s|\right), \\
(1-\mu(x)) \pi(x)+\mu(x) \xi(x) & \text { if } x \in r_{1}^{-1}\left(\text { int }|s| \backslash\left\{b_{s}\right\}\right) \backslash U_{2}, \\
\xi(x) & \text { if } x \in r_{1}^{-1}\left(\left\{b_{s}\right\}\right) .
\end{array}\right.
$$

By the properties of $\gamma$ and $\delta$, the formulas agree on the respective boundaries. So, $f$ will be well defined if we show that each pair

(a) $\pi(x)$ and $\xi(x)$,

(b) $r \circ f^{\prime} \circ \eta(x)$ and $\pi(x)$, and furthermore, $(1-\delta) r \circ f^{\prime} \circ \eta(x)+\delta \pi(x)$ and $\xi(x)$ for $0 \leq \delta \leq 1$,

(c) $H\left(f^{\prime} \circ \eta(x), \gamma(x)\right)$ and $\xi(x)$

belongs to a simplex of $M(K, n)$ for a respective $x \in r_{1}^{-1}$ (int $\left.|s| \backslash\left\{b_{s}\right\}\right)$.

To show (a), find $\tau \in(\partial s)^{(1)} \subset M(K, n)$ with $\pi(x) \in|\tau|$. Let $\hat{\tau} \in M(K, n)$ be the simplex that consists of the vertices of $\tau$ and all the vertices of the form $\left(b_{s}, m\right), d \leq m \leq n$; in particular $\tau \leq \hat{\tau}$. By the definition of $\xi$, we have that $\pi(x), \xi(x) \in|\hat{\tau}|$.

Sublemma 4.7. For $x \in r_{1}^{-1}$ (int $\left.|s|\right)$, let $\left\{v_{0}=\left(b_{\sigma_{0}}, m_{0}\right), \ldots, v_{p}=\left(b_{\sigma_{p}}, m_{p}\right)\right\} \subset V$, where $\sigma_{0} \leq \cdots \leq \sigma_{p}$ and $m_{0}<\cdots<m_{p}$, be the smallest simplex of $M\left(K^{(1)}, n\right)$ containing $x$. Then

(i) $(\exists 0 \leq q \leq p-1)\left[\sigma_{i} \in(\partial s)^{(1)} \Longleftrightarrow 0 \leq i \leq q\right]$, 
(ii) $\left(b_{\sigma_{p}}, m_{p}\right) \in S$ and $m_{p} \geq \operatorname{dim}\left(\sigma_{p}\right)=d$,

(iii) if $\eta(x)$ is defined, then $\eta(x) \in\left\{v_{0}, \ldots, v_{q}\right\}$,

(iv) $\xi(x)$ is an element of the simplex $\left\{\left(b_{s}, \max \left(d, m_{q+1}\right)\right), \ldots,\left(b_{s}, \max \left(d, m_{p}\right)\right)\right\}$.

To justify (b), assume that $x \in r_{1}^{-1}$ (int $\left.|s| \backslash\left|\overline{\operatorname{st}}\left(b_{s}, K^{(2)}\right)\right|\right)$. With $\tau$ as above and $\sigma_{q}$ as in Sublemma 4.7(i), we have $\left|\sigma_{q}\right| \subset|\tau|$. It follows that $r_{1}(\eta(x)) \in$ $\left|\sigma_{q}\right| \subset|\tau|$ (see Sublemma 4.7(iii)) and, by Lemma 4.6(iv) applied to $f^{\prime}$, we obtain $r\left(f^{\prime}(\eta(x))\right) \in|\tau|$. This shows that $r \circ f^{\prime} \circ \eta(x), \pi(x) \in|\tau| \subset|\hat{\tau}|$ and, by the proof of item $(\mathrm{a}),(1-\delta) r \circ f^{\prime} \circ \eta(x)+\delta \pi(x), \xi(x) \in|\hat{\tau}|$.

To show (c), assume $x \in U_{1}$. By Sublemma 4.7(iii), $\eta(x) \in\left|M^{m_{q}}\left(K_{d-1}^{(1)}, n\right)\right|$. Applying Lemma 4.3(vii) to $f^{\prime}$ (with $\eta(x)$ and $k=m_{q}$ ), for every $v=\left(b_{\sigma}, m\right) \in$ $\operatorname{Vert}\left(M\left(K_{d-1}, n\right)\right)$ with $t_{v}\left(f^{\prime}(\eta(x))\right) \neq 0$, either $m=\operatorname{dim}(\sigma)$ or $m \leq m_{q}$. Let $\sigma^{\prime}$ be the smallest simplex of $M\left(K_{d-1}, n\right)$ such that $H\left(f^{\prime} \circ \eta(x), \gamma(x)\right) \in\left|\sigma^{\prime}\right|$. Take any vertex $\left(b_{\tau}, m\right)$ of $\sigma^{\prime}$; note that $\tau \in \partial s$. By Lemma 4.3(vii), if $m>\operatorname{dim}(\tau)$, then $m \leq m_{q}$. It then follows that $m \leq \max \left(\operatorname{dim}(\tau), m_{q}\right)<\max \left(d, m_{q+1}\right)$. Enlarge $\sigma^{\prime}$ to the simplex $\hat{\sigma}^{\prime}$ by adding to the vertices of $\sigma^{\prime}$ all the vertices of the form $\left(b_{s}, k\right)$, where $\max \left(d, m_{q+1}\right) \leq k \leq n$. Taking into account Sublemma 4.7(iv), it is now clear that $H\left(f^{\prime} \circ \eta(x), \gamma(x)\right), \xi(x) \in\left|\hat{\sigma}^{\prime}\right|$.

Now, we verify the continuity of $f$. By the continuity of all involved maps in the formula for $f, f$ is continuous on $r_{1}^{-1}$ (int $|s| \backslash\left\{b_{s}\right\}$ ). Therefore, it is enough to show that $f$ is continuous at $x_{0}$ in the following three cases: $x_{0} \in r_{1}^{-1}\left(b_{s}\right), x_{0} \in$ $r_{1}^{-1}(|\partial s|) \backslash|\partial s|$, and $x_{0} \in|\partial s|$. Let $\left\{x_{i}\right\}_{i=1}^{\infty} \subset r_{1}^{-1}\left(\right.$ int $\left.|s| \backslash\left\{b_{s}\right\}\right)$ with $\lim x_{i}=x_{0}$.

If $x_{0} \in r_{1}^{-1}\left(b_{s}\right)$, then $\lim \mu\left(x_{i}\right)=1$ because $\lim r_{1}\left(x_{i}\right)=b_{s}$. Consequently, $\lim f\left(x_{i}\right)=\lim \left[\left(1-\mu\left(x_{i}\right)\right) \pi\left(x_{i}\right)+\mu\left(x_{i}\right) \xi\left(x_{i}\right)\right]=\xi\left(x_{0}\right)=f\left(x_{0}\right)$. If $x_{0} \in r_{1}^{-1}(|\partial s|) \backslash$ $|\partial s|$, then $\lim \gamma\left(x_{i}\right)=0, \lim \mu\left(x_{i}\right)=0$, and $\lim \eta\left(x_{i}\right)=\eta\left(x_{0}\right)=x_{0}\left(\right.$ provided $\eta\left(x_{i}\right)$ is well defined). Consequently,

$$
\begin{aligned}
& \lim H\left(f^{\prime}\left(\eta\left(x_{i}\right)\right), \gamma\left(x_{i}\right)\right)=H\left(f^{\prime}\left(\eta\left(x_{0}\right)\right), 0\right)=f^{\prime}\left(\eta\left(x_{0}\right)\right)=f^{\prime}\left(x_{0}\right)=f\left(x_{0}\right), \\
& \lim f\left(x_{i}\right)=\lim \left[\left(1-\mu\left(x_{i}\right)\right) H\left(f^{\prime}\left(\eta\left(x_{i}\right)\right), \gamma\left(x_{i}\right)\right)+\mu\left(x_{i}\right) \xi\left(x_{i}\right)\right]=f\left(x_{0}\right) .
\end{aligned}
$$

Let $x_{0} \in|\partial s|$. The following family, consisting of sets:

$$
O(W, \lambda)=\left\{(1-t) y+t z \in r^{-1}(|s|) \mid y \in W, z \in r^{-1}(|s|), 0 \leq t \leq \lambda\right\}
$$

where $W$ runs over all neighborhoods of $x_{0}$ in $r^{-1}(|\partial s|)$ and $0<\lambda<1$, is a base of neighborhoods of $x_{0}$ in $r^{-1}(|s|)$. Fix such a neighborhood $O(W, \lambda)$ of $x_{0}$. (Below, we evaluate maps at $x$ only if they are well defined at such an $x$.) The continuity of $\pi$ and the facts that $\pi\left(x_{0}\right)=x_{0}$ and $\lim _{x \rightarrow x_{0}} H\left(f^{\prime}(\eta(x)), \gamma(x)\right)=x_{0}$ imply that there exists a neighborhood $P$ of $x_{0}$ in $r_{1}^{-1}(|s|)$ such that $H\left(f^{\prime}(\eta(x)), \gamma(x)\right), \pi(x) \in$ $W$ provided $x \in P$. On the other hand, since $r\left(f^{\prime}\left(\eta\left(x_{0}\right)\right)\right)=x_{0}$ and $\pi\left(x_{0}\right)=x_{0}$, we can assume that $(1-\delta) r\left(f^{\prime}(\eta(x))\right)+\delta \pi(x) \in W$ for every $x \in P$ and every $0 \leq \delta \leq 1$. We can also assume that for $x \in P, \mu(x) \leq \lambda$ because $\mu\left(x_{0}\right)=0$. Now, for $x \in P,(1-\mu(x)) \pi(x)+\mu(x) \xi(x),(1-\mu(x)) H\left(f^{\prime}(\eta(x)), \gamma(x)\right)+\mu(x) \xi(x)$, 
and $(1-\mu(x))\left[(1-\delta(x)) r\left(f^{\prime}(\eta(x))\right)+\delta(x) \pi(x)\right]+\mu(x) \xi(x)$ belong to $O(W, \lambda)$. This completes the proof of the continuity of $f$.

Now, we verify properties (i), (ii), (iii), and (iv) of Lemma 4.6 and (vi). We need to consider a point $x \in r_{1}^{-1}$ (int $\left.|s|\right)$ only. If, additionally, $x \notin U_{2}$, then $r(f(x))=r_{1}(x)$. By the inductive assumption, $d\left(r(f(y)), r_{1}(y)\right)<\varepsilon$ for all $y \in$ $r^{-1}(|\partial s|)$; by the continuity of the maps involved, this inequality holds for all $x$ in some neighborhood of $r_{1}^{-1}(|\partial s|)$ in $r_{1}^{-1}(|s|)$. Taking $U_{2}$ to be contained in that neighborhood, we see that $d\left(r(f(x)), r_{1}(x)\right)<\varepsilon$ for all $x$. This shows (i). If $x \in$ int $|s|, x \neq b_{s}$, then $f(x)=(1-\mu(x)) \pi(x)+\mu(x) b_{s}=r_{1}(x)=x$; if $x=b_{s}$, then $f(x)=\xi\left(b_{s}\right)=b_{s}$. This shows (ii).

To show (iii), let $x \in r_{1}^{-1}$ (int $\left.|s|\right) \backslash\left|M^{k}\left(K^{(1)}, n\right)\right|, 0 \leq k \leq n$. Using the notation of Sublemma 4.7, $m_{p}>k$; by Sublemma 4.7(ii), $v_{p} \in S$ and $\max \left(m_{p}, d\right)=$ $m_{p}>k$. Now, looking at Sublemma 4.7(iv), for the vertex $v=\left(b_{s}, \max \left(m_{p}, d\right)\right)$, we have $t_{v}(\xi(x)) \neq 0$; consequently, $\xi(x) \notin\left|M^{k}\left(K^{(1)}, n\right)\right|$. This, together with the fact that $\mu(x)>0$, yields $f(x) \notin\left|M^{k}\left(K^{(1)}, n\right)\right|$ because $\xi(x)$ shows up in all the formulas defining $f(x)$.

To show (iv), fix $\sigma \in K^{(1)}$ and $x \in r_{1}^{-1}(|\sigma|) \cap r_{1}^{-1}$ (int $\left.|s|\right)$. Using the notation of Sublemma 4.7, we have $\sigma_{p} \leq \sigma$. This implies that both $r(\xi(x))=b_{s}$ and $r(\pi(x))=\pi(x)$ belong to $|\sigma|$, and $r_{1}(\eta(x)) \in|\sigma| \cap|\partial s|$ (use Sublemma 4.7(iii)). By an application of Lemma 4.6(iv) to $f^{\prime}$, we infer that $r\left(f^{\prime} \circ \eta(x)\right) \in|\sigma| \cap$ $|\partial s| \subset|\sigma|$. Since $r\left(H\left(f^{\prime} \circ \eta(x), \gamma(x)\right)\right)=r\left(f^{\prime} \circ \eta(x)\right)$, we obtain $H\left(f^{\prime} \circ \eta(x)\right.$, $\gamma(x)) \in|\sigma|$. (All maps are evaluated at $x$ if they are defined at such an $x$.) Finally, using the fact that $r$ is simplicial and looking at the formulas that define $f$, we conclude that $r(f(x)) \in|\sigma|$.

To show property (vi), let $x \in \mid M^{k}\left(K^{(1)}, n\right) \cap r_{1}^{-1}$ (int $\left.|s|\right), 0 \leq k \leq n$. We will examine $v=\left(b_{\sigma}, m\right) \in \operatorname{Vert}(M(K, n))$ for which $t_{v}(f(x)) \neq 0$ and $m>\operatorname{dim}(\sigma)$. Using the notation of Sublemma 4.7, by (iii), we obviously have $m_{p} \leq k$ and $\eta(x) \in\left|M^{k}\left(K_{d-1}, n\right)\right|$. Now, as in the proof of item (c), by an application of (vi) to $f^{\prime}$ and Sublemma 4.7(vii), we infer that if $t_{v}\left(H\left(f^{\prime} \circ \eta(x), \gamma(x)\right)\right) \neq 0$, then $m \leq$ $k$. On the other hand, by Sublemma 4.7(iv), we see that if $t_{v}(\xi(x)) \neq 0$, then $m \leq$ $\max \left(m_{p}, d\right) \leq m_{p} \leq k$ (see Sublemma 4.7(ii)). Finally, since $t_{v}(f(x)) \neq 0$ implies either $t_{v}\left(H\left(f^{\prime} \circ \eta(x), \gamma(x)\right)\right) \neq 0$ or $t_{v}(\xi(x)) \neq 0$, we complete the proof by noting that in both these cases $m \leq k$.

4.1.2. The compacta $Z_{n}$ and the retractions $\rho_{n}: Z_{n} \rightarrow\left|K_{n}\right|$. To the complex $K_{n}$, we assign the finite complex

$$
M_{n}=M\left(K^{\left(k_{n}\right)}, n\right)
$$

for a certain $k_{n}>k_{n-1}$ (which will be provided later), and we write

$$
r_{n}=r\left(K_{n}^{\left(k_{n}\right)}, n\right): M_{n}=M\left(K^{\left(k_{n}\right)}, n\right) \longrightarrow K_{n}^{\left(k_{n}+1\right)}=\left(K_{n}^{\left(k_{n}\right)}\right)^{(1)} .
$$


The set $C_{\sigma}$. For a simplex $\sigma \in K_{n}^{\left(k_{n}\right)}$ (not just $\sigma \in K_{n}^{\left(k_{n}+1\right)}$ ), we let

$$
D_{\sigma}=r_{n}^{-1}(|\sigma|), \quad C_{\sigma}=D_{\sigma} \times[0,1]
$$

We write $[\sigma, x, t]$ for a typical point of $(x, t) \in D_{\sigma} \times[0,1]=C_{\sigma}$ and identify $D_{\sigma} \ni$ $x \rightarrow[\sigma, x, 0] \in C_{\sigma}$. Write $p_{n}: C_{\sigma} \rightarrow D_{\sigma}$ for the obvious retraction given by

$$
p_{n}([\sigma, x, t])=x \quad \forall x \in D_{\sigma}, 0 \leq t \leq 1
$$

The space $Z_{n}$. We let

$$
Z_{n}=\left|M_{n}\right| \cup \bigcup\left\{C_{\sigma} \mid \sigma \in K_{n}^{\left(k_{n}\right)}\right\},
$$

where $C_{\sigma}$ are attached to $\left|M_{n}\right|$ in such a way that $C_{\sigma} \cap C_{\tau}=D_{\sigma} \cap D_{\tau}$ for $\sigma \neq \tau$. Clearly, $Z_{n}$ is a finite-dimensional metric compactum; we let $d_{n} \leq 1$ to be a compatible metric on $Z_{n}$. Now, $p_{n}$ gives rise to a retraction $\rho_{n}: Z_{n} \rightarrow\left|M_{n}\right|$ defined by

$$
\rho_{n}=r_{n} \circ p_{n}: Z_{n} \longrightarrow\left|K_{n}\right|
$$

The map $x \rightarrow v\left(K_{n}^{\left(k_{n}\right)}, n\right)(x), x \in\left|M_{n}\right|$, gives rise to a map $v_{n}=v\left(K_{n}^{\left(k_{n}\right)}, n\right) \circ p_{n}$ : $Z_{n} \rightarrow[0,1]$ so that

$$
v_{n}([\sigma, x, t])=v\left(K_{n}^{\left(k_{n}\right)}, n\right)(x) \quad \forall x \in D_{\sigma}, 0 \leq t \leq 1
$$

It follows from the definition of $v_{n}$ that $v_{n}|| M_{n} \mid=0$. Finally, we define $\gamma_{n}: Z_{n} \rightarrow$ $[0,1]$ so that $\gamma_{n}|| M_{n} \mid=0$ and $\gamma_{n}([\sigma, x, t])=t$.

The sequences $\left\{k_{n}\right\}$ and $\left(\varepsilon_{n}\right)$. Now, we inductively specify the sequences $\left\{k_{n}\right\}$ and $\left(\varepsilon_{n}\right)$ that will be crucial in verifying the countable dimensionality of $Z$. Suppose we have defined the spaces $Z_{1}, \ldots, Z_{n}$ (hence, the maps $\rho_{n}, v_{n}$, and $\gamma_{n}$ are also known) and the bonding maps $\pi_{m}^{n}: Z_{n} \rightarrow Z_{m}$ for $1 \leq m \leq n$, and that we have already chosen $k_{1}<\cdots<k_{n}(n \geq 1)$. For every set $S \subset\left|K_{n}\right|$, we have $\rho_{n}^{-1}(S) \cap$ $v_{n}^{-1}(0) \cap \gamma_{n}^{-1}(0)=S$. This, together with the fact that $\sup \left\{\operatorname{diam}(|s|) \mid s \in K_{n}^{(k)}\right\} \rightarrow$ 0 if $k \rightarrow \infty$, guarantees the existence of $k_{n+1}>k_{n}$ and $0<\varepsilon_{n} \leq 1$ such that

$$
\operatorname{diam}_{d_{m}}\left[\pi_{m}^{n}\left(\rho_{n}^{-1}\left(B\left(|\sigma|, \varepsilon_{n}\right)\right) \cap v_{n}^{-1}\left(\left[0, \varepsilon_{n}\right]\right) \cap \gamma_{n}^{-1}\left(\left[0, \varepsilon_{n}\right]\right)\right)\right] \leq 2^{-n}
$$

for all $\sigma \in K_{n}^{\left(k_{n+1}\right)}$ and $m=1, \ldots, n$. (Here, $B\left(|\sigma|, \varepsilon_{n}\right)$ stands for the generalized closed ball around $|\sigma|$ of radius $\varepsilon_{n}$.) 
To start the induction, set $k_{1}=0$. Then $K_{1}^{\left(k_{1}\right)}=K_{1}$ and $M_{1}=M\left(K_{1}, 1\right)$. Define $\rho_{1}, v_{1}$, and $\gamma_{1}$. Since, for every set $S \subset\left|K_{1}\right|, \rho_{1}^{-1}(S) \cap v_{1}^{-1}(0) \cap \gamma_{1}^{-1}(0)=S$, and since $\sup \left\{\operatorname{diam}(|s|) \mid s \in K_{1}^{(k)}\right\} \rightarrow 0$ if $k \rightarrow \infty$, there exist $k_{2}>k_{1}=0$ and $0<\varepsilon_{1} \leq$ $1 / 2$ such that $\operatorname{diam}_{d_{1}}\left[\rho_{1}^{-1}\left(B\left(|\sigma|, \varepsilon_{1}\right)\right) \cap v_{1}^{-1}\left(\left[0, \varepsilon_{1}\right]\right) \cap \gamma_{1}^{-1}\left(\left[0, \varepsilon_{1}\right]\right)\right] \leq 1 / 2$ for every $\sigma \in K_{1}^{\left(k_{2}\right)}$.

4.1.3. The bonding maps $\pi_{n}^{n+1}: Z_{n+1} \rightarrow Z_{n}$. Together with $M_{n}=M\left(K_{n}^{\left(k_{n}\right)}, n\right)$ and $M_{n+1}=M\left(K_{n+1}^{\left(k_{n+1}\right)}, n+1\right)$, and maps $r_{n}=r\left(K_{n}^{\left(k_{n}\right)}, n\right)$ and $r_{n+1}=r\left(K_{n+1}^{\left(k_{n+1}\right)}, n+1\right)$, we also consider

$$
M\left(K_{n}^{\left(k_{n}+1\right)}, n\right), \quad \hat{r}_{n}=r\left(K_{n}^{\left(k_{n+1}\right)}, n\right): M\left(K_{n}^{\left(k_{n+1}\right)}, n\right) \longrightarrow K_{n}^{\left(k_{n+1}+1\right)} .
$$

The simplicial "bonding" map $q_{n}: K_{n+1} \rightarrow K_{n}$ gives rise to the simplicial map

$$
q_{n}^{\left(k_{n+1}\right)}: K_{n+1}^{\left(k_{n+1}\right)} \longrightarrow K_{n}^{\left(k_{n+1}\right)} .
$$

This, in turn, yields a simplicial map

$$
\mu_{n}: M^{n}\left(K_{n+1}^{\left(k_{n+1}\right)}, n+1\right)=M_{n+1}^{n} \longrightarrow M\left(K_{n}^{\left(k_{n+1}\right)}, n\right)
$$

defined as follows:

$$
\mu_{n}\left(\left(b_{\sigma}, m\right)\right)=\left(b_{q_{n}^{\left(k_{n+1}\right)}(\sigma)}, m\right) \quad \text { for }\left(b_{\sigma}, m\right) \in \operatorname{Vert}\left(M_{n+1}^{n}\right) .
$$

The following functions between complexes will be used throughout the remaining part of this text:

$$
\begin{aligned}
& K_{n+1}^{\left(k_{n+1}\right)} \ni \sigma \longrightarrow \sigma^{\prime}=q_{n}^{\left(k_{n+1}\right)}(\sigma) \in K_{n}^{\left(k_{n+1}\right)}, \\
& K_{n+1}^{\left(k_{n+1}\right)} \ni \sigma \longrightarrow \hat{\sigma} \in K_{n}^{\left(k_{n}\right)}, \quad\left|\sigma^{\prime}\right| \subset|\hat{\sigma}|,
\end{aligned}
$$

and $\hat{\sigma}$ being the smallest simplex with this property.

Lemma 4.8. For every $\sigma \in K_{n+1}^{\left(k_{n+1}\right)}, \mu_{n}\left(r_{n+1}^{-1}(|\sigma|) \cap M_{n+1}^{n}\right) \subset \hat{r}_{n}^{-1}\left(\left|\sigma^{\prime}\right|\right)$.

Proof. Assume that $x \in\left|M_{n+1}^{n}\right|$ with $r_{n+1}(x) \in|\sigma|$, where $\sigma \in K_{n+1}^{\left(k_{n+1}\right)}$. Then, $x=$ $\sum\left\{t_{v}(x) v \mid v=\left(b_{\tau}, m\right), m \leq n\right\}$ (here $\left(b_{\tau}, m\right)$ are the vertices of the smallest simplex of $M_{n+1}^{n}$ containing $\left.x\right)$. It follows that, for each such vertex $\left(b_{\tau}, m\right)$, we have $\tau \leq \sigma$. By the definition of $\mu_{n}, \mu_{n}(x)=\sum_{v} t_{v}(x)\left(b_{q_{n}^{\left(k_{n+1}\right)}(\tau)}, m\right)$. Consequently, $\hat{r}_{n}\left(\mu_{n}(x)\right)=\sum_{v} t_{v}(x) b_{q_{n}^{\left(k_{n+1}\right)}(\tau)}$. Hence, $\hat{r}\left(\mu_{n}(x)\right) \in\left|\sigma^{\prime}\right|$.

Using the fact that for every $\sigma, \tau \in K_{n+1}^{\left(k_{n+1}\right)}, \sigma \neq \tau$, we have that $C_{\sigma} \cap C_{\tau}=$ $r_{n+1}^{-1}(\sigma) \cap r_{n+1}^{-1}(\tau)$, and that $\hat{r}_{n}^{-1}\left(\left|\sigma^{\prime}\right|\right)$ is contractible (see Corollary 4.4(i)); by Lemma 4.8 , we extend $\mu_{n}|| M_{n+1}^{n} \mid$ over the whole $Z_{n+1}$ so that

$$
\left(\forall \sigma \in K_{n+1}^{\left(k_{n+1}\right)}\right) \quad\left[\mu_{n}\left(C_{\sigma}\right) \subset \hat{r}_{n}^{-1}\left(\left|\sigma^{\prime}\right|\right)\right] .
$$


We need a "subdivision map" $f_{n}:\left|M\left(K_{n}^{\left(k_{n+1}\right)}, n\right)\right| \rightarrow\left|M_{n}\right|=\left|M\left(K_{n}^{\left(k_{n}\right)}, n\right)\right|$ such that

$$
\left(\forall s \in K_{n}^{\left(k_{n+1}\right)}\right) \quad\left[f_{n}\left(\hat{r}_{n}^{-1}(|s|)\right) \subset r_{n}^{-1}(|s|)\right]
$$

we achieve this by applying Lemma 4.6 with $K=K_{n}^{\left(k_{n}\right)}, l=k_{n+1}-k_{n}$, and $\varepsilon=\varepsilon_{n}$. Now, we can define

$$
\theta_{n}=f_{n} \circ \mu_{n}: Z_{n+1} \longrightarrow\left|M_{n}\right| \subset Z_{n}
$$

Now, we extend $\theta|| M_{n+1}^{n} \mid$ to the desired map $\pi_{n}^{n+1}$. For $\sigma \in K_{n+1}^{\left(k_{n+1}\right)}$, consider the sets $C_{\sigma} \subset Z_{n+1}$ and $C_{\hat{\sigma}} \subset Z_{n}$ (for which we have $D_{\sigma}=r_{n+1}^{-1}(|\sigma|)$ and $D_{\hat{\sigma}}=$ $r_{n}^{-1}(|\hat{\sigma}|)$, respectively). Let

$$
E_{\sigma}^{n+1}=C_{\sigma} \backslash\left|M_{n+1}^{n}\right|
$$

Applying (4.31) and (4.32) (with $\left.s=\sigma^{\prime} \in K_{n}^{\left(k_{n+1}\right)}\right)$, we infer that $f_{n}\left(\mu_{n}\left(C_{\sigma}\right)\right) \subset$ $r_{n}^{-1}\left(\left|\sigma^{\prime}\right|\right) \subset r_{n}^{-1}(|\hat{\sigma}|)$ because $\left|\sigma^{\prime}\right| \subset|\hat{\sigma}|$. We conclude that $\theta_{n}\left(C_{\sigma}\right) \subset r_{n}^{-1}(|\hat{\sigma}|)=$ $D_{\hat{\sigma}}$. Letting $\beta_{n+1}: Z_{n} \rightarrow\left[0, \varepsilon_{n}\right]$ with $\beta_{n+1}^{-1}(0)=\left|M_{n+1}^{n}\right|$ be a continuous steering function, it is easy to see that the following formula well defines the required bonding map $\pi_{n}^{n+1}: Z_{n+1} \rightarrow Z_{n}$ :

$$
\pi_{n}^{n+1}(x)= \begin{cases}\theta_{n}(x) & \text { if } x \in\left|M_{n+1}^{n}\right| \\ {\left[\hat{\sigma}, \theta_{n}(x), \beta_{n+1}(x)\right]} & \text { if } x \in \bar{E}_{\sigma}^{n+1}, \sigma \in K_{n+1}^{\left(k_{n+1}\right)}\end{cases}
$$

Moreover, we have

$$
\pi_{n}^{n+1}\left(C_{\sigma}\right) \subset C_{\hat{\sigma}}
$$

We put $\pi_{m}^{n+1}=\pi_{m}^{n} \circ \pi_{n}^{n+1}$ and $\pi_{n+1}^{n+1}=\mathrm{id}$.

We show condition (4.2) of Step 2, that is, $\pi_{n}^{n+1}\left(\left|K_{n+1}\right|\right) \supset\left|K_{n}\right|$. To this end, for a given $s \in K_{n}$, we find $\sigma \in K_{n+1}$ such that $|s|=\pi_{n}^{n+1}(|\sigma|)$. Since $q_{n}$ is surjective, there exists $\sigma \in K_{n+1}, \operatorname{dim}(\sigma)=\operatorname{dim}(s)$, with $q_{n}(\sigma)=s$. Hence, the maps $q_{n} \mid \sigma$ and $q_{n}^{\left(k_{n+1}+1\right)}: \sigma^{\left(k_{n+1}+1\right)} \rightarrow s^{\left(k_{n+1}+1\right)}$ are simplicial isomorphisms. We identify $K_{n+1}^{\left(k_{n+1}+1\right)}$ with a subcomplex of $M_{n+1}$, and we have that $\sigma^{\left(k_{n+1}+1\right)} \subset M_{n+1}^{n}$ because $\operatorname{dim}(\sigma)=\operatorname{dim}(s) \leq \operatorname{dim} K_{n} \leq n$. By the definition of $\mu_{n}, \mu_{n} \mid \sigma^{\left(k_{n+1}+1\right)}=$ $q_{n}^{\left(k_{n+1}+1\right)} \mid \sigma^{\left(k_{n+1}+1\right)}$. So, using Lemma 4.6(i), $\pi_{n}^{n+1}(|\sigma|)=f_{n}\left(\mu_{n}(|\sigma|)\right)=\mu_{n}(|\sigma|)=$ $\left|q_{n}^{\left(k_{n+1}+1\right)}(\sigma)\right|=|\sigma|$. This shows condition (4.2) of Step 2 .

As announced earlier, the compactum $Z$ is defined as

$$
Z=\lim _{(}\left(Z_{n}, \pi_{n}^{n+1}\right)
$$


4.1.4. The countable dimensionality of $Z$. Here are some additional properties of $\pi_{n}^{n+1}$ that are needed to prove the countable dimensionality of $Z$.

Lemma 4.9. The following holds:

(i) (for all $0 \leq k \leq n-1)\left[\left(\pi_{n}^{n+1}\right)^{-1}\left(\left|M_{n}^{k}\right|\right) \subset\left|M_{n+1}^{k}\right|\right]$,

(ii) $\left(\right.$ for all $\left.\sigma \in K_{n+1}^{\left(k_{n+1}\right)}\right)($ for all $0 \leq m \leq n)\left[\operatorname{diam}_{d_{m}}\left(\pi_{m}^{n+1}\left(E_{\sigma}^{n+1}\right)\right) \leq 2^{-n}\right]$; consequently, $\operatorname{diam}\left(\pi_{n+1}^{-1}\left(E_{\sigma}^{n+1}\right)\right)<2^{-n+1}$,

(iii) the family $\left\{E_{\sigma}^{n+1} \mid \sigma \in K_{n+1}^{\left(k_{n+1}\right)}\right\}$ consists of open, pairwise disjoint subsets of $Z_{n+1}$.

Proof. To verify (i), take $x \in Z_{n+1} \backslash\left|M_{n+1}^{k}\right|$ for $0 \leq k \leq n-1$. If, additionally, $x \notin$ $\left|M_{n+1}^{n}\right|$, then $\beta_{n+1}(x) \neq 0$, and consequently, $\pi_{n}^{n+1}(x)=\left[\hat{\sigma}, \theta_{n}(x), \beta_{n+1}(x)\right] \in C_{\hat{\sigma}} \mid$ $\left|M_{n}\right|$ (see the definition of $\pi_{n}^{n+1}$ and (4.30) for the choice of $\hat{\sigma}$ ). Now, assume that $x \in\left|M_{n+1}^{n}\right|$. Taking into account the smallest simplex of $M_{n+1}^{n}$ that contains $x$, we have $x=\sum t_{v_{i}}(x)\left(b_{\sigma_{i}}, m_{i}\right)$, where $v_{i}=\left(b_{\sigma_{i}}, m_{i}\right)$ are the vertices of this simplex. Then, there exists $i_{0}$ such that $m_{i_{0}}>k$. Since $\mu_{n}(x)=\sum t_{v_{i}}(x)\left(b_{\sigma_{i}^{\prime}}, m_{i}\right)$ (see (4.30) for the definition of $\left.\sigma_{i}^{\prime}\right)$, we conclude that $\mu_{n}(x) \notin\left|M^{k}\left(K_{n}^{\left(k_{n+1}\right)}, n\right)\right|$. Finally, by Lemma 4.6(iii),

$$
\pi_{n}^{n+1}(x)=\theta_{n}(x)=f_{n}\left(\mu_{n}(x)\right) \notin\left|M^{k}\left(K_{n}^{\left(k_{n}\right)}, n\right)\right|
$$

To verify (ii), let $x \in E_{\sigma}^{n+1}$ for $\sigma \in K_{n+1}^{\left(k_{n+1}\right)}$. Then, by the definition of $\pi_{n}^{n+1}$,

$$
\pi_{n}^{n+1}(x)=\left[\hat{\sigma}, f_{n}\left(\mu_{n}(x)\right), \beta_{n+1}(x)\right] .
$$

We show that $\pi_{n}^{n+1}(x) \in \rho_{n}^{-1}\left(B\left(\left|\sigma^{\prime}\right|, \varepsilon_{n}\right) \cap v_{n}^{-1}\left(\left[0, \varepsilon_{n}\right]\right) \cap \gamma_{n}^{-1}\left(\left[0, \varepsilon_{n}\right]\right)\right)$ (see $(4.30)$ for the definition of $\sigma^{\prime}$ ); this will yield (ii) via an application of (4.25) (to the case of $m=n$ ).

We recall that $\beta_{n+1}(x) \leq \varepsilon_{n}$. By the definition of $\gamma_{n}$, we have

$$
\gamma_{n}\left(\pi_{n}^{n+1}(x)\right)=\gamma_{n}\left(\left[\hat{\sigma}, f_{n}\left(\mu_{n}(x)\right), \beta_{n+1}(x)\right]\right)=\beta_{n+1}(x) \leq \varepsilon_{n}
$$

and by (4.24) and Lemma 4.6(v), $v_{n}\left(\pi_{n}^{n+1}(x)\right)=v\left(K_{n}^{\left(k_{n}\right)}, n\right)\left(f_{n}\left(\mu_{n}(x)\right)\right)<\varepsilon_{n}$ (the "subdivision map" $f_{n}$ was found for $\left.\varepsilon=\varepsilon_{n}\right)$. It remains to show that $\rho_{n}\left(\pi_{n}^{n+1}(x)\right) \in$ $B\left(\left|\sigma^{\prime}\right|, \varepsilon_{n}\right)$. By $(4.21)$,

$$
\rho\left(\pi_{n}^{n+1}(x)\right)=r_{n}\left(p_{n}\left(\left[\hat{\sigma}, f_{n}\left(\mu_{n}(x)\right), \beta_{n+1}(x)\right]\right)\right)=r_{n}\left(f_{n}\left(\mu_{n}(x)\right)\right) .
$$

Since, by Lemma 4.6(ii), $d\left(r_{n}\left(\mu_{n}(x)\right), \hat{r}\left(\mu_{n}(x)\right)\right)<\varepsilon_{n}$, we will be done if only $\hat{r}_{n}\left(\mu_{n}(x)\right) \in\left|\sigma^{\prime}\right|$; but this is a consequence of (4.31) and the fact that $C_{\sigma} \supset E_{\sigma}^{n+1}$ (see (4.36)). 
Let $z, z^{\prime} \in \pi_{n+1}^{-1}\left(E_{\sigma}^{n+1}\right)$, that is, $z=\left(\pi_{m}(z)\right)$ and $z^{\prime}=\left(\pi_{m}\left(z^{\prime}\right)\right)$. Using the fact that $\pi_{n+1}(z), \pi_{n+1}\left(z^{\prime}\right) \in E_{\sigma}^{n+1}$, we can estimate

$$
\begin{aligned}
d_{Z}\left(z, z^{\prime}\right) & =\sum_{m=1}^{\infty} 2^{-m} d_{m}\left(\pi_{m}(z), \pi_{m}\left(z^{\prime}\right)\right) \\
& =\sum_{m=1}^{n} 2^{-m} d_{m}\left(\pi_{m}(z), \pi_{m}\left(z^{\prime}\right)\right)+\sum_{m=n+1}^{\infty} 2^{-m} d_{m}\left(\pi_{m}(z), \pi_{m}\left(z^{\prime}\right)\right) \\
& \leq \sum_{m=1}^{n} 2^{-m} d_{m}\left(\pi_{m}^{n+1}\left(\pi_{n+1}(z)\right), \pi_{m}^{n+1}\left(\pi_{n+1}\left(z^{\prime}\right)\right)\right)+2^{-n} \\
& \leq\left(\sum_{m=1}^{n} 2^{-m} 2^{-n}\right)+2^{-n}<2 \cdot 2^{-n}=2^{-n+1} .
\end{aligned}
$$

Item (iii) easily follows from Corollary 4.4(ii) and the fact that $C_{\sigma} \cap C_{\tau}=$ $D_{\sigma} \cap D_{\tau}$ for $\sigma \neq \tau$.

According to the definition of $Z_{n}$, for every $n \geq 1$,

$$
Z_{n}=\left|M_{n}^{n-1}\right| \cup \bigcup\left\{E_{\sigma}^{n} \mid \sigma \in K_{n}^{\left(k_{n}\right)}\right\}
$$

recall that $E_{\sigma}^{n}=C_{\sigma} \backslash\left|M_{n}^{n-1}\right|$. It follows that $Z=P_{0} \cup \bigcup_{n=1}^{\infty} P_{n}$, where

$$
P_{0}=\bigcap_{n=1}^{\infty} \pi_{n}^{-1}\left(\bigcup\left\{E_{\sigma}^{n} \mid \sigma \in K_{n}^{\left(k_{n}\right)}\right\}\right), \quad Y_{n}=\pi_{n}^{-1}\left(\left|M_{n}^{n-1}\right|\right) .
$$

It is enough to show that $\operatorname{dim}\left(P_{n}\right)<\infty$ for $n=0,1, \ldots$

Since, for every $n$, the space $P_{0}$ is covered by the family $\left\{\pi_{n}^{-1}\left(E_{\sigma}^{n}\right) \mid \sigma \in K_{n}^{\left(k_{n}\right)}\right\}$, which consists of open and pairwise disjoint sets of diameter less than $2^{-n+2}$ (see Lemma 4.9(ii) and (iii)), we conclude that $\operatorname{dim}\left(P_{0}\right) \leq 0$.

On the other hand, we show that $\operatorname{dim}\left(P_{n}\right) \leq n-1$ for $n \geq 1$. Since in this case $P_{n}=\pi_{n}^{-1}\left(\left|M_{n}^{n-1}\right|\right)$, we have that $P_{n}=\underline{\lim }\left(\pi_{m}\left(P_{n}\right), \pi_{m}^{k} \mid \pi_{k}\left(P_{n}\right)\right)$. Now, it is enough to show that $\pi_{m}\left(P_{n}\right) \subset\left|M_{m}^{n-1}\right|$ for all $m \geq n$. To this end, take $z \in P_{n}=$ $\pi_{n}^{-1}\left(\left|M_{n}^{n-1}\right|\right)$; so, $\pi_{n}(z) \in\left|M_{n}^{n-1}\right|$. Since $\pi_{n}^{m}\left(\pi_{m}(z)\right)=\pi_{n}(z)$, it follows that $\pi_{m}(z)$ $\in\left(\pi_{n}^{m}\right)^{-1}\left(\left|M_{n}^{n-1}\right|\right) \subset\left|M_{m}^{n-1}\right|$; the last inclusion is a consequence of Lemma 4.9(i).

4.2. The map $\varphi: Z \rightarrow X$. This section provides details for the statements of Step 3.

For every $z \in Z$ and $n \geq 1$, let $\sigma_{n}(z)$ be the smallest simplex $\sigma_{n}(z) \in K_{n}^{\left(k_{n}\right)}$ with $\pi_{n}(z) \in C_{\sigma_{n}(z)}$ and let $s_{n}(z)$ be the smallest simplex of $K_{n}$ such that $\left|\sigma_{n}(z)\right| \subset$ $\left|s_{n}(z)\right|$, that is, $s_{n}(z)$ is the smallest simplex of $K_{n}$ such that $\rho_{n}\left(\pi_{n}(z)\right) \in\left|s_{n}(z)\right|$. We set

$$
F_{n}(z)=\bigcap_{\alpha \in s_{n}(z)} \bar{V}_{\alpha}
$$


Clearly, we have that $F_{n}(z)$ is a compact subset of $X$, and

$$
\operatorname{diam}\left(F_{n}(z)\right) \leq \Delta_{n}
$$

(see (K4) for the definition of $\Delta_{n}$ ).

Lemma 4.10. For every $1 \leq n \leq m, x \in F_{n}(z)$, and $y \in F_{m}(z), d_{\|\cdot\|}(x, y) \leq 2 \Delta_{n}$.

Proof. First, we verify that for all $1 \leq n<m, s_{n}(z) \leq q_{n, m}\left(s_{m}(z)\right)$, where $q_{n, m}=$ $q_{n} \circ q_{n+1} \circ \cdots \circ q_{m-1}$. Actually, we can assume that $m=n+1$. Assign to $z$ the simplex $\sigma_{n+1}(z) \in K_{n+1}^{\left(k_{n+1}\right)}$ accordingly, and write briefly $\sigma_{n+1}(z)=\sigma$. Using (4.30), consider $\hat{\sigma} \in K_{n}^{\left(k_{n}\right)}$ and $\sigma^{\prime} \in K_{n}^{\left(k_{n+1}\right)}$; hence, $\left|\sigma^{\prime}\right| \subset|\hat{\sigma}|$. Since $\pi_{n+1}(z) \in C_{\sigma}$ and by (4.36), $\pi_{n}(z)=\pi_{n}^{n+1}\left(\pi_{n+1}(z)\right) \in C_{\hat{\sigma}}$. By the minimality of $\sigma_{n}(z)$, we have that $\sigma_{n}(z) \leq \hat{\sigma}$. Since $\left|\sigma_{n+1}(z)\right| \subset\left|s_{n+1}(z)\right|$, we obtain $\left|\sigma^{\prime}\right| \subset\left|q_{n}\left(s_{n+1}(z)\right)\right|$. The fact that $q_{n}\left(s_{n+1}(z)\right) \in K_{n}$ implies that $\left|\sigma^{\prime}\right| \subset|\hat{\sigma}| \subset\left|q_{n}\left(s_{n+1}(z)\right)\right|$ (by the minimality of $\hat{\sigma}$ ) and, furthermore, it implies $s_{n}(z) \leq q_{n}\left(s_{n+1}(z)\right)$ (by the minimality of $s_{n}(z)$ and the fact $\left.\left|\sigma_{n}(z)\right| \subset|\hat{\sigma}| \subset\left|q_{n}\left(s_{n+1}(z)\right)\right|\right)$.

Now, use the fact that $q_{n, m}$ is a projection to see that

$$
F_{m}(z) \subset\left\{V_{\alpha} \mid \alpha \in q_{n, m}\left(s_{m}(z)\right)\right\}
$$

By the fact that $s_{n}(z) \subset q_{n, m}\left(s_{m}(z)\right)$,

$$
F_{n}(z) \cup F_{m}(z) \subset \bigcup\left\{V_{\alpha} \mid \alpha \in q_{n, m}\left(s_{m}(z)\right)\right\}
$$

However, $q_{n, m}\left(s_{m}(z)\right)$ is a simplex; hence, the intersection of the above family is nonempty, and therefore, $d(x, y) \leq 2 \Delta_{n}$ for every $x \in F_{n}(z)$.

Applying Lemma 4.10, we see that for every $z,\left(F_{n}(z)\right)$ is a Cauchy sequence in the hyperspace of $X$. This, together with (4.46), shows that $\varphi(z)=\lim F_{n}(z)$ well defines $\varphi: Z \rightarrow X$. To verify condition (4.4a) of Lemma 4.1, let $\alpha \in A_{n}$ such that $\rho_{n}\left(\pi_{n}(z)\right) \in \operatorname{st}\left(\alpha, K_{n}\right)$. Then, $\alpha \in s_{n}(z)$ and hence $F_{n}(z) \subset \bar{V}_{\alpha}$. This, together with Lemma 4.10 , shows that $d\left(\varphi(z), \bar{V}_{\alpha}\right) \leq 3 \Delta_{n}$. This completes the proof of Lemma 4.1.

\section{References}

[1] C. Bessaga and A. Pełczyński, Selected Topics in Infinite-Dimensional Topology, Monografie Matematyczne, vol. 58, PWN-Polish Scientific Publishers, Warsaw, 1975.

[2] H. F. Bohnenblust and S. Karlin, On a theorem of Ville, Contributions to the Theory of Games, vol. 1, Annals of Mathematics Studies, no. 24, Princeton University Press, New Jersey, 1950, pp. 155-160.

[3] R. Cauty, Quelques problèmes sur les groupes contractiles et la théorie des rétractes [Some contractible group problems and the theory of retracts], Mat. Stud. 3 (1994), 111-116 (French).

[4] - Le théorème de point fixe de Lefschetz-Hopf pour les compacts ULC, preprint, 1999. 
[5] - Solution du problème de point fixe de Schauder [Solution of Schauder's fixed point problem], Fund. Math. 170 (2001), no. 3, 231-246 (French).

[6] A. Cellina and A. Lasota, A new approach to the definition of topological degree for multi-valued mappings, Atti Accad. Naz. Lincei Rend. Cl. Sci. Fis. Mat. Natur. (8) 47 (1969), 434-440.

[7] T. Dobrowolski, On extending mappings into nonlocally convex linear metric spaces, Proc. Amer. Math. Soc. 93 (1985), no. 3, 555-560.

[8] Every convex compactum has the simplicial approximation property, preprint, 2001.

[9] - Fixed-point theorem for convex-valued mappings, preprint, 2001.

[10] T. Dobrowolski and W. Marciszewski, Rays and the fixed point property in noncompact spaces, Tsukuba J. Math. 21 (1997), no. 1, 97-112.

[11] T. Dobrowolski and J. Mogilski, Regular retractions onto finite-dimensional convex sets, Function Spaces. The Second Conference (Edwardsville, Ill, 1994) (K. Jarosz, ed.), Lecture Notes in Pure and Applied Mathematics, vol. 172, Marcel Dekker, New York, 1995, pp. 85-99.

[12] T. Dobrowolski and H. Toruńczyk, On metric linear spaces homeomorphic to $l_{2}$ and compact convex sets homeomorphic to Q, Bull. Acad. Polon. Sci. Sér. Sci. Math. 27 (1979), no. 11-12, 883-887.

[13] J. Dugundji and A. Granas, Fixed Point Theory. I, Monografie Matematyczne, vol. 61, Państwowe Wydawnictwo Naukowe (PWN-Polish Scientific Publishers), Warsaw, 1982.

[14] R. Engelking, Theory of Dimensions Finite and Infinite, Sigma Series in Pure Mathematics, vol. 10, Heldermann Verlag, Lemgo, 1995.

[15] K. Fan, Fixed-point and minimax theorems in locally convex topological linear spaces, Proc. Nat. Acad. Sci. U.S.A. 38 (1952), 121-126.

[16] I. L. Glicksberg, A further generalization of the Kakutani fixed theorem, with application to Nash equilibrium points, Proc. Amer. Math. Soc. 3 (1952), 170-174.

[17] J. H. Gresham, A class of infinite-dimensional spaces. II. An extension theorem and the theory of retracts, Fund. Math. 107 (1980), no. 3, 237-245.

[18] S.-T. Hu, Theory of Retracts, Wayne State University Press, Detroit, 1965.

[19] M. Hukuhara, Sur l'existence des points invariants d'une transformation dans l'espace fonctionnel, Japan. J. Math. 20 (1950), 1-4 (French).

[20] S. Kakutani, A generalization of Brouwer's fixed point theorem, Duke Math. J. 8 (1941), 457-459.

[21] N. J. Kalton, N. T. Peck, and J. W. Roberts, An F-Space Sampler, London Mathematical Society Lecture Note Series, vol. 89, Cambridge University Press, Cambridge, 1984.

[22] V. L. Klee Jr., Some topological properties of convex sets, Trans. Amer. Math. Soc. 78 (1955), 30-45.

[23] S. Mardešić and J. Segal, Shape Theory, North-Holland Mathematical Library, vol. 26, North-Holland, Amsterdam, 1982.

[24] S. Mardešić and N. Uglešić, On irreducible mappings into polyhedra, Topology Appl. 61 (1995), no. 2, 187-203.

[25] R. D. Mauldin (ed.), The Scottish Book, Birkhäuser, Massachusetts, 1991.

[26] S. Mazur, Un théorème sur les points invariants, Ann. Soc. Math. Polon. 17 (1938), 110 (French). 
[27] R. P. Millspaugh, L. R. Rubin, and P. J. Schapiro, Irreducible representations of metrizable spaces and strongly countable-dimensional spaces, Fund. Math. 148 (1995), no. 3, 223-256.

[28] J. Schauder, Der Fixpunktsatz in Funktionalräumen, Studia Math. 2 (1930), 171-180 (German).

[29] A. Tychonoff, Ein Fixpunktsatz, Math. Ann. 111 (1935), 767-776 (German).

[30] M. Zarichnyr, Universal map of $\sigma$ onto $\Sigma$ and absorbing sets in the classes of absolute Borelian and projective finite-dimensional spaces, Topology Appl. 67 (1995), no. 3, 221-230.

Tadeusz Dobrowolski: Department of Mathematics, Pittsburg State University, Pittsburg, KS 66762, USA

Current address: Department of Mathematics, University of Missouri-Columbia, Columbia, MO 65211, USA

E-mail address: tdobrowo@mail.pittstate.edu 


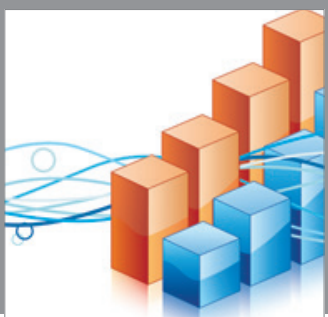

Advances in

Operations Research

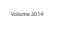

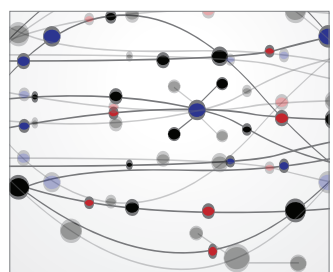

\section{The Scientific} World Journal
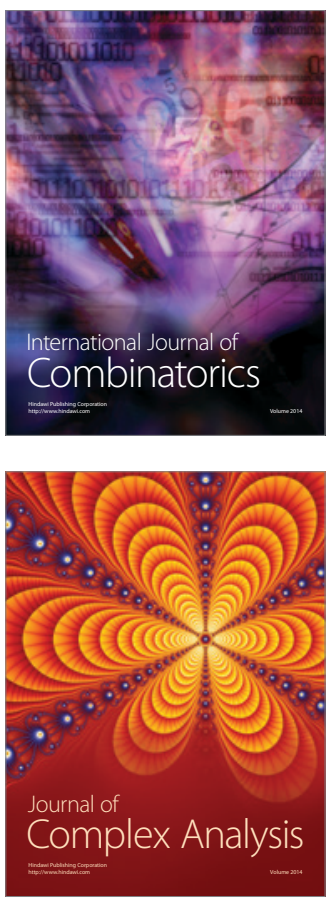

International Journal of

Mathematics and

Mathematical

Sciences
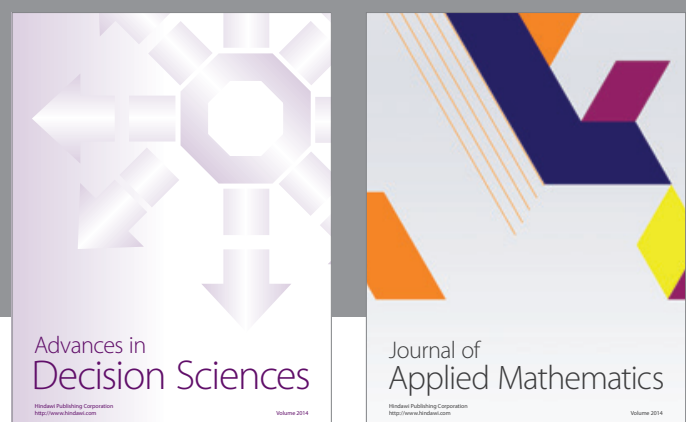

Journal of

Applied Mathematics
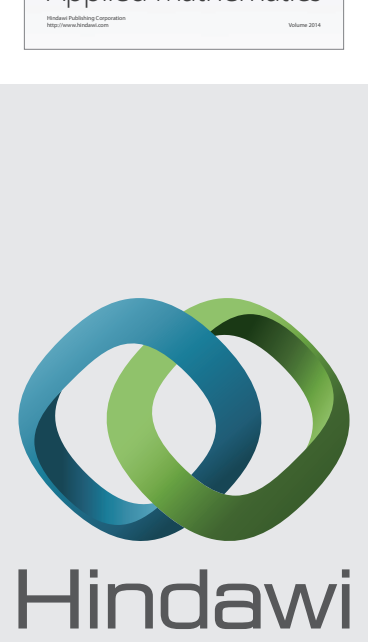

Submit your manuscripts at http://www.hindawi.com
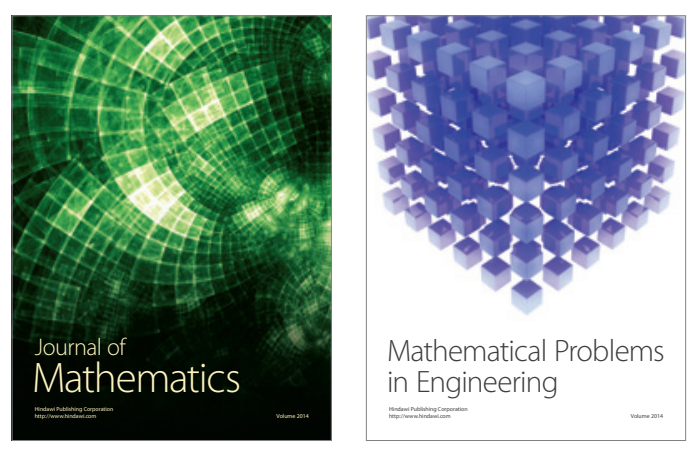

Mathematical Problems in Engineering
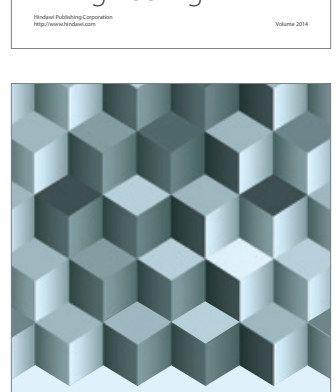

Journal of

Function Spaces
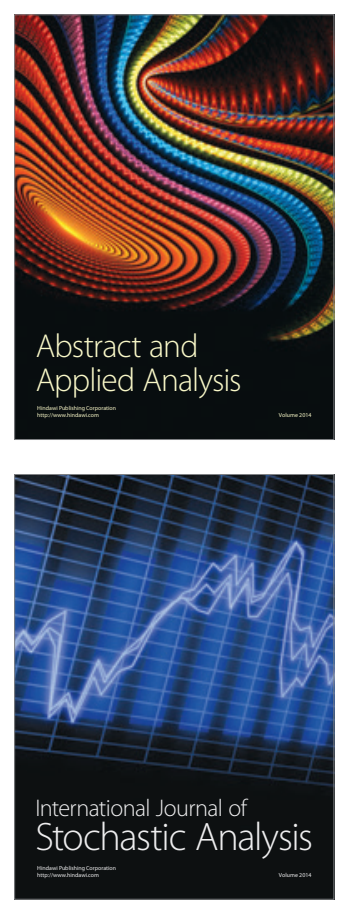

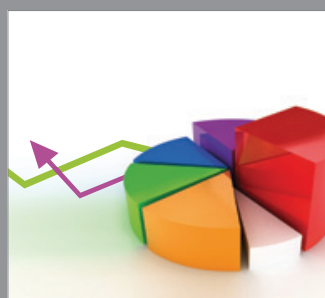

ournal of

Probability and Statistics

Promensencen
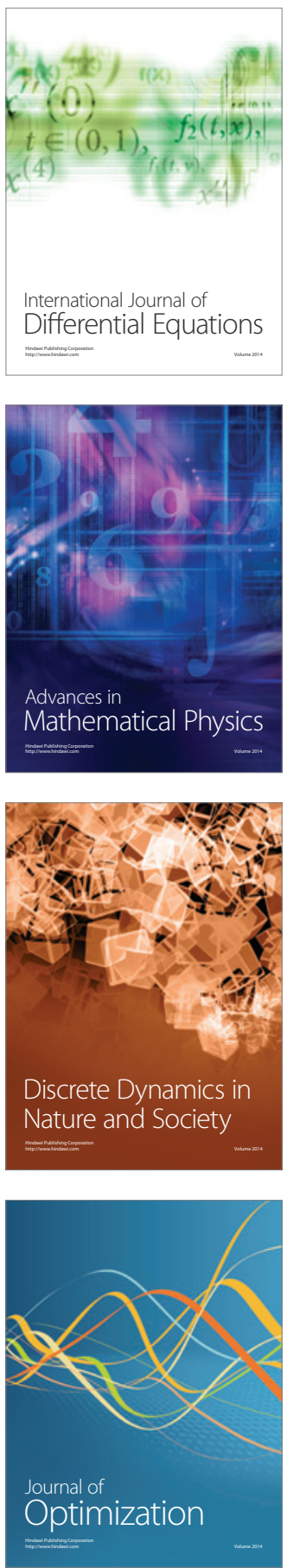